\title{
LES TRENTE PREMIERS VERSETS \\ DE LA LITANIE D'OSIRIS À ESNA (ESNA 217)*
}

\author{
PAR \\ CHRISTIAN LEITZ \\ Ägyptologisches Institut der Universität Tübingen \\ Schloß Hohentübingen \\ D-72070 Tübingen
}

L'état des recherches sur les litanies d'Esna n'a pratiquement pas changé depuis la mort de Serge Sauneron en 1976 : six ans après le tragique accident, son manuscrit a été publié par les soins de J. J. Clère, devenant le huitième volume de la série Esna. Comme Clère le relève dans son avant-propos, Sauneron en avait entièrement rédigé le texte principal, mais il manquait une révision et une harmonisation finales du manuscrit. Au cours des vingtcinq dernières années, quelques versets ont été cités, traduits et parfois commentés ici et là, mais d'une manière générale, ces litanies sont considérées comme relevant d'un jeu de l'écriture ptolémaïque, comparables aux deux hymnes à Khnoum, écrits presque exclusivement avec des hiéroglyphes figurant des béliers ou des crocodiles.

Le début de la litanie d'Osiris est gravé sur la colonne 3, laquelle date du règne de Domitien (81-96) ; la fin est située sur la colonne 2, gravée un peu plus tard, sous le règne de Trajan (98-117). A priori, ce texte est facile à lire, tout comme celui des autres litanies; il est établi que les trois premiers signes, après le $n$ datif, se lisent Wsìr, ou plus exactement, il s'agit des trois consonnes $w, s$ et $r$. Les épithètes - habituellement entre une et trois - sont écrites conventionnellement et posent rarement des problèmes de lecture.

Sauneron parle d'une véritable alchimie hiéroglyphique dont il n'a trouvé que très peu d'exemples ailleurs, où, selon ses propres mots, «dans l'orthographe des noms divins doivent pouvoir se retrouver toutes les épithètes possibles de la divinité, et comme les éléments essentiels servant à écrire les actes ou les attitudes qu'on lui prête. Autrement dit, l'explication par calembour ne porte pas sur le nom dans son ensemble, ni même sur le son individuel des consonnes qui le composent, mais sur les possibilités graphiques offertes par

\footnotetext{
* Les thèmes abordés dans cet article ont fait l'objet de deux conférences à l'École Pratique des Hautes Études, section des Sciences religieuses, à l'invitation de Christiane Zivie-Coche, qu'il m'est agréable de remercier pour cet honneur et pour son hospitalité pendant mon séjour parisien. Ivan Guermeur a bien voulu corriger le texte français de mes quatre conférences à la Sorbonne mais aussi celui de cette contribution pour la $R d E$; qu'il trouve ici mes remerciements les plus sincères. Cette étude - quoique déjà assez longue - n'est qu'un rapport préliminaire d'un projet beaucoup plus vaste qui abordera toutes les litanies à Esna ; un projet dont l'achèvement, du fait des multiples obligations de l'auteur, n'est envisagée que dans quelques années.
}

Revue d'Égyptologie 59, 231-266. doi: 10.2143/RE.59.0.2036291

Tous droits réservés @ Revue d'Égyptologie, 2008. 
les signes qui servent à l'écrire. LE JEU DE MOTS N'EST PLUS PHONÉTIQUE, MAIS ORTHOGRAPHIQUE, et il ne peut être sensible qu'à celui qui lit ces textes». Sauneron avait pu isoler sept cas principaux de ces jeux extraordinairement complexes et subtils ${ }^{1}$.

Pour ma part, j'ai essayé, dans un premier temps, de ne travailler qu'avec trois catégories : une graphique, une phonétique et une autre allégorique (cette dernière étant plus ou moins la même que le sixième cas de Sauneron). Comme souvent, ce premier essai se révéla peu concluant; il est en effet parfois difficile d'opérer des distinctions entre les différentes catégories, un même phénomène pouvant assez souvent appartenir à deux catégories. Il faut d'abord remarquer qu'on ne peut se contenter, simplement, d'établir les possibles relations existant entre l'écriture du nom divin et ses épithètes (le texte en clair). Bien au contraire, la plupart des textes et des allusions qui en découlent sont, au premier abord, absolument incompréhensibles. Sauneron a, bien entendu, essayé de déterminer la structure sous-jacente des litanies et les a comparées avec des arétalogies grecques d'Isis. Toutefois, il a fini par confesser que «ici, rien de semblable. Nous avons relu maintes fois, verset après verset, les diverses litanies, avec l'espoir de déceler quelque disposition subtile dont la trame nous aurait d'abord échappé, en vain ${ }^{2}$.

Finalement, j'en suis arrivé à la conclusion que l'on pouvait expliciter tous les phénomènes avec un classement en cinq catégories : A) purement graphique ; B) une catégorie graphique/phonétique ; C) une catégorie purement phonétique ; D) une catégorie phonétique/allégorique ; et enfin, E) une catégorie purement allégorique. La catégorie A) peut être subdivisée en deux groupes : soit on fait des allusions avec exactement le même signe, soit on utilise un signe apparenté à l'autre. Dans la terminologie de Sauneron, il s'agit des catégories 1,2 et 7 . Chacune des cinq catégories se combine librement avec un élément du même verset $x$ ou du suivant $x+1$ ou encore du verset $x+2$; il s'agit, en principe, de la clef qui permet la compréhension du texte. La seule litanie d'Osiris occupe, dans Esna VIII, trois pages et demie de hiéroglyphes tandis qu'il en faut vingt-et-une pour reproduire en typographie l'ensemble des litanies ; mon commentaire, qui ne traite que des trente premier versets de la litanie d'Osiris, ne fait déjà pas moins de quarante pages et ceci n'en est pas la fin.

Verset (1): rmm T3-sny «À Osiris-Onnophris, juste de voix, roi des dieux, le grand dieu, qui réside à Esna».

A. Catégorie graphique (1b) : le lièvre (SS) dans le texte d'accompagnement renvoie au même signe qui se trouve au verset suivant, dans l'écriture de Sokar-Osiris.

${ }^{1}$ S. Sauneron, L'écriture figurative dans les textes d'Esna (Esna VIII), 1982, p. 56-58.

${ }^{2}$ Esna VIII, 13. 
A. Catégorie graphique (1b) : C. Catégorie phonétique (1a) : la fleur de lotus (

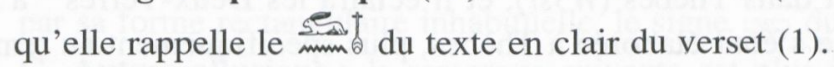

C. Catégorie phonétique (1a-b) : Isis et Nephthys associées ( de snty «les deux sœurs». Elles renvoient ainsi au groupe snt (mm employé dans l'écriture de $T 3$-sny.

C. Catégorie phonétique (2a) : l'allusion se retrouve dans la fleur de lotus ( (Wenge qui se lit aussi $s \check{n}$, «lotus». On obtient donc, par calembour, une allusion au de l'écriture de T3-sny:

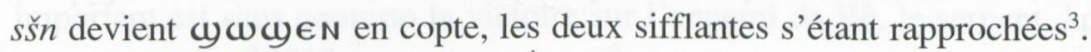

C. Catégorie phonétique (2b) : évoque le figurant dans l'écriture de Sokar-Osiris au verset (2).

D. Catégorie phonétique/allégorique (1a-b) : l'allusion qui suit fonctionne avec l'enfant sur la fleur de lotus ( lotus») ; autrement dit, on obtient un renvoi au du texte d'accompagnement et au verset (2). Par ailleurs, l'enfant solaire sur la fleur de lotus ( évoque aussi le mythe de la naissance d'Osiris, dont une des descriptions les plus détaillées figure au temple d'Opet à Karnak ${ }^{5}$ : «Lorsqu'elle (la déesse 'Ipt-wrt) se hâta <vers> le temple de 'Ipt-wrt, elle accoucha (hms.s) ${ }^{6}$ d'Onnophris, juste de voix, en tant qu'aîné de Geb (ou comme première étoile). La lumière $(\check{s} w)$ se lève sur la terre ainsi que sur ses frères, dans leur lieu de naissance (st-mshnt); ils sont à côté de lui, dans sa place et Nout devient le nom du ciel et ses enfants deviendront ses étoiles ${ }^{7}$. La ${ }^{8}$ lumière $(\check{s} w)$ brille dans tout le nome thébain $(W 3 s t)$ et dans le temple de 'Iptwrt ; la couronne blanche est sur sa tête et l'uraeus l'entoure, Sa Majesté est un bel adolescent (hwn nfr); on l'appela wn (peut-être enfant) au commencement (m-ḩ3t), au moment où la lumière (wny) parut sur sa brique de naissance $(m s h n t . f)$; il ouvrit $(w n)$ la matrice $(n f r t)^{9}$ (?) dès le début $\left(m \breve{s}^{\circ}\right) \ldots$ ? $\ldots^{10}$, avant ses frères : ainsi le nomma-t-on Onnophris, juste de voix, lorsqu'il fut créé à Thèbes $(W 3 s t)$.» Un autre texte se trouve dans le voisinage du précédent, au bandeau du soubassement ${ }^{11}$ : «En ce jour parfait qui se produisit à Thèbes (Nît),

${ }^{3}$ C'est-à-dire que le c s'est assimilé, au voisinage d'un $\omega$, à $\boldsymbol{y}^{\circ}$ : cf. M.-L. Ryhiner, L'offrande du Lotus (Rites égyptiens VI), 1986, p. 3.

${ }^{4}$ Esna VIII, p. $115, \mathrm{n}^{\circ} 16$.

5 Opet I, p. 183 (le début de la partie traduite ici se trouve à la colonne 3, côté sud) ; cf. la traduction de C. De Wit, Les inscriptions du temple d'Opet à Karnak, III (BAe 13), 1968, p. 101-102 et son commentaire p. 146; voir également Fr.-R. Herbin, Le livre de parcourir l'éternité (OLA 58), 1994, p. 176-177 et Id., RdE 54 (2003), p. 106.

${ }^{6}$ Ce sens de hms n'est pas relevé par le Wörterbuch, mais la traduction proposée par De Wit me semble concluante à cet endroit.

7 Voir A. Gutbub, Textes fondamentaux de la théologie de Kom Ombo (BdE 47), 1973, p. 330, n. g'.

${ }^{8}$ Début du montant nord.

${ }^{9}$ Cf. De Wit avec renvoi à Opet $\mathrm{I}, 186,1$ (là, le déterminatif est un $\smile$ ).

${ }^{10} \mathrm{La}$ lecture $(\%, \underline{h} n m)$ et la traduction de ne me paraissent pas clairs, la proposition de De Wit («encerclant [= protégeant $]$ les cœurs de ses frères») me semble peu convaincante.

11 Opet I, 186 ; voir Fr.-R. Herbin, RdE 54 (2003), p. 106.

RdE 59 (2008) 
le beau ${ }^{12}$, qui est apparu à Karnak ('Ipt-swt), est venu ; sa parole est sortie alors que la lumière était dans l'CEil de Rê ('Irt- $\mathrm{R}^{\mathrm{e}}$, une désignation de Thèbes) et dès que la matrice fut ouverte dans $B n n t^{13}$, Osiris apparut dans Thèbes (W3st), et il éclaira les Deux-Terres ${ }^{14}$ à travers l'obscurité» ${ }^{15}$. On relèvera que s'était élaborée à Thèbes, plus spécifiquement au temple $\mathrm{d}^{\prime}$ Opet $^{16}$, le lieu de la naissance d'Osiris, une tradition qui combinait la venue au monde d'Osiris avec l'apparition de la lumière et qui créait des étiologies du nom d'Osiris avec la racine $w n$; l'enfant sur la fleur de lotus de notre premier verset, qui peut aussi se lire $w n$, fait référence à cette tradition.

E. Catégorie allégorique (1a) : la fleur de lotus ( de la Haute Égypte ${ }^{17}$; elle fait écho à la plante $\succsim$ qui représente habituellement l'Égypte méridionale.

E. Catégorie allégorique (1b) : il faut peut-être encore relever une autre allusion en rapport avec l'enfant sur la fleur de lotus ( En effet, en Égypte, et plus particulièrement à l'époque ptolémaïque et romaine, on trouve figuré non seulement un enfant sur la fleur de lotus, mais aussi souvent un serpent (par exemple Harsomtous) ${ }^{18}$. On pourrait donc également voir ici un renvoi au serpent ( $00 \Omega \Omega$ ) qui figure dans le nom de Sokar-Osiris du verset (2).

F. Relation avec la litanie entière (1a) : le signe de l'enfant sur la fleur de lotus (wigurant au début de la litanie, débute idéalement ce texte: la naissance d'Osiris se fait en analogie avec celle du dieu solaire ${ }^{19}$, né tantôt à la première heure du jour, tantôt au premier jour de l'année ${ }^{20}$. Comme je m'efforcerai de le montrer, d'autres exemples permettent d'établir un système selon lequel la litanie entière peut être comparée à une année cultuelle (comparer les versets [8] et [12]) et un autre où les vingt-quatre premiers versets peuvent être mis en balance avec les douze heures du jour et les douze heures de la nuit.

(2) mm A. Catégorie graphique (1b) : le groupe $\sum^{\infty}$ renvoie naturellement au même groupe dans le verset (3).

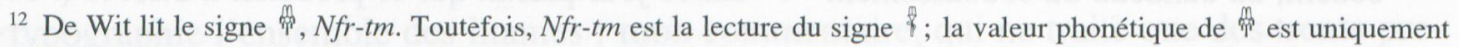
$n f r$, d'où ma traduction. J'y vois un calembour renvoyant, avec la lumière (wny), à $W n(n)-n f r$, comme le wn figurant dans le dernier montant.

${ }^{13}$ Le nom du temple de Khonsou à Karnak, cf. D. Mendel, Die kosmogonischen Inschriften in der Barkenkapelle des Chonstempels von Karnak (MRE 9), 2003, p. 80-81.

${ }^{14} t 3 w y$ est écrit avec 舟忽, ce qui constitue aussi un jeu de signe avec deux hiéroglyphes qui se lisent habituellement $h p r$ «advenir».

15 On trouve, au temple d'Opet, d'autres allusions qui lient la naissance et le nom d'Onnophris à l'origine de la lumière : C. De Wit, op. cit., p. 146-147 ; Fr.-R. Herbin, Le livre de parcourir l'éternité (OLA 58), 1994, p. 165 ; $176-177$.

${ }^{16}$ Comparer également avec le verset (41) de la litanie d'Osiris.

17 Voir les remarques de P. Wilson, A Ptolemaic Lexikon (OLA 78), 1997, p. 929 au sujet de la plante sšnt (jonc ou lotus?).

18 Cf. M.-L. Ryhiner, op. cit., p. 183-184.

19 E. Brunner-Traut, in LÄ IV, col. 1092, s.v. «Lotos». wnw «enfant» peut être une désignation du jeune dieu solaire (Chr. Leitz [éd.], LGG II, 389c).

20 Cf. Chr. Leitz, Tagewählerei. Das Buch ḥßt nḥ̣ pḥ.wy d̆ und verwandte Texte (ÄgAbh 55), 1994, p. 13. 


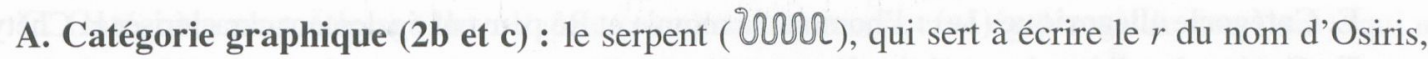
fait écho aux serpents ( $(\bullet$ ) des versets (3) et (4); le signe $\longleftarrow$, utilisé pour écrire $t 3$, évoque, par sa forme rectangulaire inhabituelle, le signe cim du verset (3).

G. Autres allusions : la remarque suivante est plus intéressante. On a vu au verset (1) que l'enfant sur la fleur de lotus (造) peut être une manifestation du dieu solaire au matin; au verset (3), on voit l'homme âgé qui s'appuie sur son bâton (䧄) qui pourrait, lui, être une évocation d'Atoum, le démiurge solaire à l'heure du coucher. Ceci rappelle le modèle défini par J. Assmann ${ }^{21}$, qui consiste à diviser en trois phases la course journalière du soleil. Il y manque le midi, lequel doit se trouver au verset (2). Dans ce modèle, l'évènement le plus important est sans conteste la victoire sur l'ennemi de Rê, le serpent Apophis ${ }^{22}$. On supposera que le serpent ( $200 \Omega \Omega$ ) peut aussi être une manifestation d'Apophis. Mais, on peut aller encore un peu plus loin dans l'explication; en effet, avant le hiéroglyphe figurant un serpent, on reconnaît un groupe est un verbe signifiant «battre» et Wns est un toponyme qui se situe dans le $\mathrm{XIX}^{\mathrm{e}}$ nome de Haute Égypte (Oxyrhynquite). Cette localité est connue depuis le Moyen Empire; elle figure sur la Chapelle Blanche de Sésostris $\mathrm{I}^{\mathrm{er}}$. Whs est une des villes du dieu Seth ${ }^{24}$, lequel est parfois aussi appelé $W n s y$ «celui de la ville $W n s »$; or, il est bien établi que Seth, à la Basse Époque, est pratiquement identifié au serpent Apophis. En conclusion, on pourrait admettre que $s k r$ wns, «battre celui de la ville Wns», c'est-à-dire Seth, évoque Apophis et dès lors, considérer que le serpent ( $200 \Omega \Omega$ ), figurant après wns, joue le rôle d'un déterminatif du nom de Seth ou bien d'Apophis ${ }^{25}$.

(3) mm

A. Catégorie graphique (1b) : il est assez aisé de reconnaître les jeux graphiques : le signe de Rê ( $(Q)$ ) figure à deux reprises au verset (4).

A. Catégorie graphique (2b) : l'homme âgé qui s'appuie sur un bâton (䦽) renvoie à l'homme qui tient son bâton (惯) au verset (4).

D. Catégorie phonétique/allégorique (1a) : l'allusion suivante est plus subtile : le groupe P $\mathrm{b}$ : peut se lire $\operatorname{snn} R^{e}$ «l'image de Rê», qui est aussi une désignation d'Osiris et de Sokar-Osiris ${ }^{27}$.

${ }^{21}$ Cf. J. Assmann, in LÄ V, col. 1087-1090, s.v. «Sonnengott» (son modèle des quatre phases ou, en cas d'identité de la phase III avec la phase IV, des trois phases de la course journalière du soleil).

${ }_{22}$ Cf. J. Assmann, Re und Amun. Die Krise des polytheistischen Weltbilds im Ägypten der 18.-20. Dynastie (OBO 51), 1983, p. 71-82.

${ }^{23}$ Les graphies de $s k r r$ écrites $\mathcal{J}^{-\infty}$ ne posent pas de problème, cf. Chr. Leitz (éd.), LGG VI, 655b-c.

24 Cf. Chr. Leitz (éd.), $L G G$ II, $417 \mathrm{~b}$ avec d'autres renvois.

${ }^{25}$ En passant, je relève que cette allusion à la localité $W n s$, située dans le XIX ${ }^{\mathrm{e}}$ nome de Haute Égypte, se trouve précisément à la colonne 19. Les produits du hasard étant rares dans ce texte, il est loisible de supposer que le choix de cette position est délibéré. Le $\mathrm{n}^{\circ} 19$ attribué à cette colonne est de toute évidence correct, puisque sur les dix-huit premières colonnes est gravée la litanie consacrée à Neith.

26 À propos du tombeau (štyt) d'Osiris(-Sokaris) à Héliopolis, cf. Fr.-R. Herbin, RdE 54 (2003), p. 94.

27 Chr. Leitz (éd.), LGG VI, 389a. 
E. Catégorie allégorique (1a) : l'homme, la momie et Rê peuvent également caractériser la Chétyt ${ }^{28}$. E. Catégorie allégorique (1a) : l'usage d'une pierre $(\square)$ comme déterminatif de štyt - à la place du déterminatif de la maison $(\square \square)$ - n'est pas rare. Puisque le papyrus du Delta (III, 1-2) précise que la štyt d'Héliopolis est bâtie en pierre éternelle ( $3 t \underline{d t}$ ), on peut admettre que l'emploi de ce déterminatif fait référence à ce matériau privilégié, employé au tombeau d'Osiris à Héliopolis ${ }^{29}$.

E. Catégorie allégorique (1b-c) : l'homme âgé est également attesté comme une manifestation d'Atoum, dieu d'Héliopolis. Celui-ci constitue donc un renvoi au signe de Rê (\&Q), utilisé à deux reprises dans le nom d'Osiris du verset (4), lequel est également dieu solaire à Héliopolis. Par ailleurs, il constitue un renvoi supplémentaire à Chou et Tefnout, la deuxième génération d'Héliopolis, les deux enfants d'Atoum.

(4) mm $\stackrel{0}{0}$ (désignation d'Esna)».

A. Catégorie graphique (1b) : le disque solaire entouré par le cobra ( $\bullet$ ) se retrouve également sur la tête de Tefnout (4i au verset (5).

D. Catégorie phonétique/allégorique (1a) : on peut déterminer une allusion phonétique/allégorique qui fonctionne avec Rê ( $\bullet \bullet$ ), le dieu solaire d'Héliopolis, $H \otimes$, 'Iwnw en égyptien,

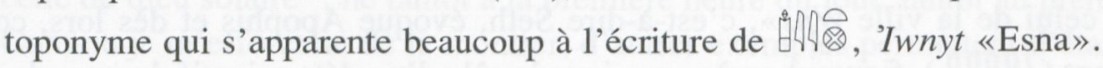

E. Catégorie allégorique (1a): la lune (๑) qui figure dans le nom d'Osiris renvoie assez clairement à l'épithète $G_{0} / h^{\sharp}$, iwn $w r$ «le grand pilier $^{30} »$, désignation fréquente de la lune ${ }^{31}$, qu'il peut à l'occasion porter.

(5) mm la voie de Routy (le double-lion, manifestation de Chou et de Tefnout)».

A. Catégorie graphique (1a) : le premier jeu graphique est aisé à remarquer; signe du chemin (굴 figure dans le nom d'Osiris et dans le texte en clair. Il s'agit du premier cas que Sauneron a mentionné dans son commentaire.

A. Catégorie graphique (2a) : le deuxième jeu graphique se retrouve dans l'hiéroglyphe avec les deux jambes, qu'on retrouve aussi comme déterminatif de $w 3 t$ «chemin» ( $(\Omega)$.

C. Catégorie phonétique (2a) : le premier jeu de cette catégorie se cache dans l'hiéroglyphe de la langue de bœuf ( $)$, qui se lit imy-r3 (mr) «préposé»; le lion $\$$ D dans le texte d'accompagnement peut aussi avoir la même lecture.

${ }^{28}$ Voir Chr. Leitz (éd.), LGG V, 348b (Hry-ib-štyt = Atoum); V, 848b (Hnty-hnnw-n-štyt = Atoum); V, 365c (Hrynmit.f-m-štyt.f $=$ Osiris) et VIII, $309 \mathrm{~b}$ (5 épithètes de Rê en relation avec la Chétyt).

29 Cf. D. Meeks, Mythes et légendes du Delta d'après le papyrus Brooklyn 47.218.84 (MIFAO 125), 2006, p. 6, 57 et surtout p. 183-184.

${ }^{30}$ La référence à Esna dans Chr. Leitz (éd.), LGG I, 195b doit être classée à la page 196a.

${ }^{31}$ Chr. Leitz (éd.), LGG I, 193 c (Iwn) et 195b (Iwn-wr). 
C. Catégorie phonétique (2b) : l'autre jeu phonétique concerne également le lion (\$D), qui a une deuxième lecture, $n b$ «maître». Celle-ci fait écho au cobra dressé ( ( $)$ ) du verset suivant, qui peut aussi se lire $n b$.

E. Catégorie allégorique (1b) : je suggère de reconnaître ici deux allusions allégoriques ; chacune est liée aux jumeaux Chou et Tefnout (㤔通). Plusieurs textes de Kom Ombo et d'Esna désignent Chou et Tefnout comme les deux oisillons de $R \hat{e}^{32}$, c'est-à-dire que les deux dieux de la fin du verset (5) font allusion à $\underline{t} 3 w y\left(\frac{2}{\psi}\right)$, la ville des deux oisillons, mentionnée à la fin du verset (6).

E. Catégorie allégorique (1c) : dans le second cas, Chou et Tefnout (何解) renvoient à la déesse crocodilocéphale qui allaite deux jeunes crocodiles ( châteaux», du verset (7), qui est rarement attestée. Un texte d'Esna, hymne adressé à Neith (Esna III, 252, 28) $)^{33}$, est très informatif à ce propos : «Tu (c'est-à-dire Neith) es la maîtresse d'Esna, la protection ${ }^{34}$ de la campagne mystérieuse, au nord de la butte des deux oisillons, celle qui a allaité les deux crocodiles en leur nom de Chou et Tefnout, qui veille ses châteaux (c'est la déesse crocodilocéphale de notre verset) ${ }^{35}$, qui tient embrassé les deux crocodiles ${ }^{36}$ dans ses bras - c'est-à-dire Rê et Osiris, les deux oisillons de son fils Rê dans la maison de Sahourê, et qui fournit les offrandes divines des dieux et des déesses». À la lecture de cette partie de l'hymne à Neith, l'allusion paraît claire: les deux divinités Chou et Tefnout renvoient à euxmêmes dans leur forme de petits crocodiles.

F. Relation avec la litanie entière (1a): on peut aller au-delà dans l'analyse de ce verset. Jusqu'ici, je n'ai pas évoqué la voie de Routy, le double-lion, or, celle-ci est connue par une composition attestée de la XVIII ${ }^{\mathrm{e}}$ dynastie à l'époque ptolémaïque : le soi-disant chapitre $15 \mathrm{~B}$ III du Livre des morts. Ce texte est en réalité un hymne au soleil couchant ${ }^{37}$ comme son titre le précise : «Adorer Rê au soir, lorsqu'il se couche dans la montagne $b 3 h w$ » Rê y est nommé à plusieurs reprises Rê-Atoum. Un petit passage indique: shd. voie de Rosetau (une partie du monde souterrain), après que tu as ouvert la voie de Routy ${ }^{38}$ :

${ }^{32}$ Chr. Leitz (éd.), LGG VII, 448c avec plusieurs références à Kom Ombo et Esna. Comparer aussi avec Esna III, 252, $28(\S 6)$.

33 Traduction de S. Sauneron, Les fêtes religieuses d'Esna aux derniers temps du paganisme (Esna V), 1962 , p. 111.

${ }^{34}$ S. Sauneron, Esna V, p. 114, n. (o), propose, grâce au parallèle d'Esna III, 216, 17 (74), de corriger ${ }^{\prime}$ en ? Puisque le nom d'Osiris au verset $(9)$ est écrit avec $\hat{R}$ et , mettant ainsi en exergue un aspect «protecteur», on peut douter - vu la rareté du signe - de la nécessité d'une telle correction. Peut-être est-il préférable de corriger $\sigma^{*}$ en 0 .

${ }^{35}$ Rst-hwwt.s est la lecture du signe (corriger Chr. Leitz [éd.], LGG IV, 699c d'après Id., Quellentexte zur ägyptischen Religion I, Die Tempelinschriften der griechisch-römischen Zeit [Einführungen und Quellentexte zur Ägyptologie 2], 2004, 116 = commentaire de l'hymne à Anoukis de Komîr, col. 26, D. Valbelle, BIFAO 83 [1983], ill. 9 après la p. 160 avec un parallèle exact au texte d'Esna).

${ }^{36}$ Pour hh-n-Nt, «gorge de Neith», comme surnom du crocodile voir D. Meeks, Mythes et légendes du Delta, p. 147, n. 513 .

37 Un commentaire détaillé de ce texte se trouve chez J. Assmann, Liturgische Lieder an den Sonnengott (MÄS 19) 1969 , p. 15-112 (à propos de «la voie de Routy», le double-lion, cf. p. 38, 1. 56 avec son commentaire p. 68); pour les deux heures de l'Amdouat, voir en dernier lieu U. Rößler-Köhler, dans R. Gundlach, W. Seipel (éd.), Das frühe ägyptische Königtum (ÄAT 36, 2), 1999, p. 73-96.

${ }^{38}$ E. g. E. Naville, Das Ägyptische Totenbuch der XVIII. bis XX. Dynastie, 1886, vol. II, 27. 
J. Assmann, dans son commentaire, fait un parallèle avec les $4^{\mathrm{e}}$ et $5^{\mathrm{e}}$ heures de $\mathrm{l}^{\prime}$ Amdouat où figurent des représentations du règne de Sokar de Rosetau, à la $4^{\mathrm{e}}$ heure et de la voie de Routy, à la $5^{\mathrm{e}}$ heure ; Routy lui-même est figuré, tel un double-lion, au registre inférieur. On est tenté de croire que, étant donné la rareté de l'expression «ouvrir la voie de Routy», son emploi au verset (5) est lié à la mention qu'on lit à la $5^{\mathrm{e}}$ heure de $\mathrm{l}^{\prime}$ 'Amdouat.

F. Relation avec la litanie entière (1a): les douze premiers versets peuvent correspondre aux douze heures du jour, les douze versets suivants aux douze heures de la nuit (de bons exemples se trouvent aux versets 17 à 19 ). Le nom de la $5^{\mathrm{e}}$ heure du jour est $N s b y t$ «la Brûlante ${ }^{39}$; on trouve une allusion à cette désignation dans l'écriture du nom d'Osiris avec

(6) mm toponyme près d'Esna) dans le District-des-deux-oisillons (une autre désignation d'Esna)».

A. Catégorie graphique (1a-c) : les deux crocodiles ( renvoient au crocodile du texte d'accompagnement, à celui qui se trouve au verset (7) et également à la déesse crocodilocéphale du verset (7).

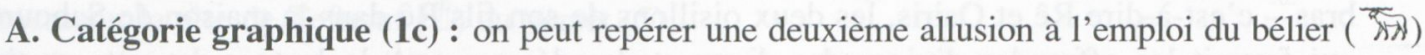
dans le nom d'Osiris aux versets (6) et (8).

A. Catégorie graphique (2b) : les deux crocodiles ( phale (

C. Catégorie phonétique (1a) : relèvent de cette catégorie plusieurs allusions qui jouent, généralement, avec les valeurs phonétiques habituelles des signes. Le cas présent est peut-être une

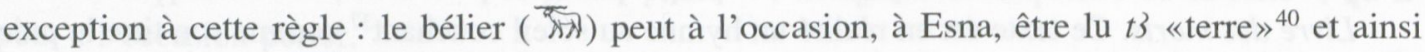
rappeler le groupe $\square \sim$ du toponyme Hnty-t3 du texte en clair.

C. Catégorie phonétique (1a-c) : le crocodile ( valeur $n b$ et renvoyer aux divers signes ayant cette valeur phonétique aux versets (6) à (8): le

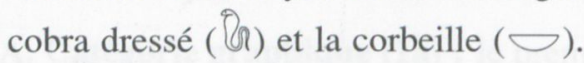

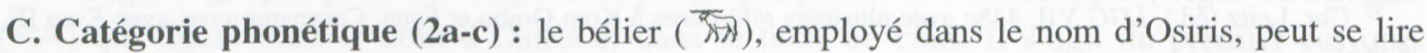
$n t r$, comme le crocodile ( bien entendu, mettre en doute cette analyse - tant de hiéroglyphes différents, particulièrement à Esna, pouvant être lus $n b$ et $n t r$.

D. Catégorie phonétique/allégorique (1a-c) : le nom d'Osiris ( hnm, c'est-à-dire Khnoum ${ }^{41}$. Il s'agit donc d'une allusion aux lieux de culte de ce dieu, lesquels sont évoqués à cinq reprises aux versets (6) à (8): Hnty-t3, T3wy, 'b3, Pr-ntrr et Pr-Hnmw $w^{42}$.

${ }^{39}$ Chr. Leitz (éd.), $L G G$ IV, 351a.

40 Cf. Chr. Leitz, SAK 29 (2001), p. 260.

${ }^{41}$ Cf. Esna VIII, p. 146 pour $=$ ha .

42 Cf. S. Sauneron, Quatre campagnes à Esna (Esna I), 1959, p. 28-29 et avec une correction en Esna V, p. 316 (Pr$n \underline{t r}$ et $\operatorname{Pr}$-Hnmw) ; Chr. Leitz (éd.), LGG V, 847b (Hnty-t3) et VII, 448c - 449a (T3wy) et Esna VI, 500, 7-8 (Khnoum-Rê $\left.n b{ }^{c} b 3\right)$. 
E. Catégorie allégorique (1a) : le bélier ( est nommé, au verset (69) de sa litanie ${ }^{43}$ : «le préposé de Hnty-t ${ }^{34}$, le bélier ( $s r$ ), à l'appel de qui vient l'inondation».

F. Relation avec la litanie entière (1a) : il est également possible de trouver, au verset (6), un élément qui établit une relation avec la litanie entière : les trois crocodiles (rॅs). Celui-ci figure dans l'emblème du sixième nome de Haute Égypte (㨫). Le verset (6) est donc le premier élément, mais pas le dernier, qui permet d'établir une relation entre les numéros des versets et l'ordre canonique des nomes égyptiens; on pourra également voir l'exemple du verset $(15)^{45}$.

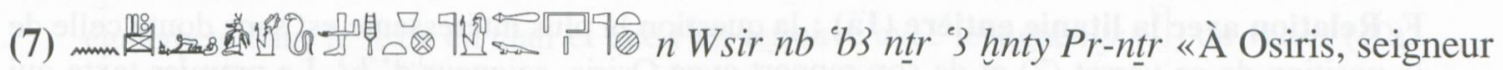
de ${ }^{c} b 3^{46}$, le grand dieu qui préside à $P r-n t r^{47}$ ».

A. Catégorie graphique (1c) : la déesse crocodilocéphale Rst-hwwt.s ( renvoie au signe qui figure la même déesse dans le nom d'Osiris du verset (9).

A. Catégorie graphique $(\mathbf{2 a - b})$ : la déesse fait également référence aux crocodiles ( des versets (7) et (8).

B. Catégorie graphique/phonétique (1b) : le caractère graphique/phonétique de l'allusion suivante est plus raffiné : l'encensoir (8), représenté dans le nom d'Osiris, évoque l'acte de faire un encensement et le petit récipient à encens, au début du signe, peut à lui seul se lire sntr , «encens, encenser». Avec cette valeur, on dispose d'une allusion à l'écriture du nom d'Osiris du verset suivant (

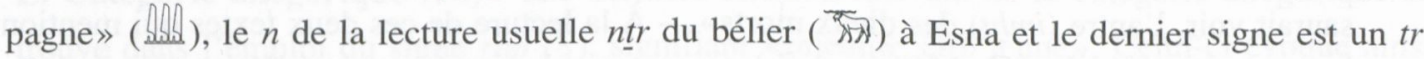
(I), les trois hiéroglyphes se lisent donc sans problème sntr.

C. Catégorie phonétique (2a) : une deuxième lecture possible pour l'autel (疋), qui figure dans le nom d'Osiris, est 'b3, qui constitue alors une allusion au toponyme 'b3, mentionné dans le texte en clair - ce que Sauneron avait déjà remarqué et que je rappelle ici par souci d'exhaustivité.

C. Catégorie phonétique (2a) : un des noms égyptiens de l'encensoir ( (258) est ntry, d'où l'on peut déduire - même si ce signe n'est pas attesté comme idéogramme - une allusion phonétique au ntr '3, «le grand dieu» et au toponyme Pr-ntr du texte en clair.

43 Esna III, 225, 22 (69).

44 D'autres références pour la relation entre Khnoum et cette localité dans Chr. Leitz (éd.), $L G G$ V, 847b.

45 On verra aussi H. Beinlich, Die «Osirisreliquien». Zum Motiv der Körperzergliederung in der altägyptischen Religion ( $\ddot{A} g A b h$ 42), 1984, p. 218-220. La relique d'Osiris du VIe nome de Haute Égypte est constituée des «membres du dieu qu'a sauvé Horus du crocodile»; aussi, dans ce texte, la mention du crocodile sert-elle à établir une relation thématique avec le signe représentant le $\mathrm{VI}^{\mathrm{e}}$ nome de Haute Égypte.

46 S. Sauneron traduit en Esna VIII, 40 «Osiris, seigneur de 'Aba» et, à un autre endroit, (Esna V, 351 à propos d'Esna III, 197, 24) «la tombe d'Osiris». Son commentaire dans Villes et légendes d'Égypte (BdE 90), 1983, p. 112-114 et dans MDAIK 16 (1958), p. 274, n. 3 montre bien qu'il s'agit toujours du même toponyme.

${ }^{47}$ On trouve un même ordonnancement des épithètes sur la colonne voisine $\mathrm{n}^{\circ} 9$, à peu près au même endroit (cf. le schéma d'Esna III, 280A). 
E. Catégorie allégorique (1a) : l'autel chargé d'offrandes (略) et l'encensoir ( sion aux offrandes pour Osiris, consacrées, précisément, à ‘b3. Le texte le plus informatif à cet égard se trouve sur une des colonnes de la façade, dans une longue invocation à Neith créatrice, peut-être en relation avec les cérémonies du 13 Épiphi (Esna II, 163, 26) : «elle (c'est-à-dire Neith) tourna son visage vers $P r-n t r$, au nord (? $)^{48}$ d'Esna. C'est un lieu saint qui n'a pas d'équivalent, pour assurer l'approvisionnement funèbre (sfsf $3 w)$ d'Osiris seigneur d' ' $b 3$, et des défunts qui sont dans sa suite». À propos des relations existant entre la déesse Rst-hwwt.s ( les offrandes et le toponyme ' $b 3$, on verra le commentaire du verset (5) et Esna III, 252, 28 (§6).

F. Relation avec la litanie entière (1a) : la question la plus intéressante est sans doute celle de la position de ce verset (7) et de son rapport avec Osiris, seigneur d' 'b3. Le premier texte qui apporte quelques éléments de réponse est une description des cérémonies de la fête du 19 Épiphi, gravée sur la colonne 1 (Esna III, 197, 24-25) ${ }^{49}$ : «l'enfer divin qui se trouve à cet endroit, c'est l'enfer mystérieux de Kematef, accompagné de Chou et Tefnout, et d'Atoum qui est enseveli avec eux. C'est l'antre des dieux morts, des septs propos $(\underline{d} 3 i s w)^{50}$ matérialisés de Méthyer en leur aspect de faucons. On les appelle 'dieux antérieurs'. On nomme (cet antre) le château des $B a(h w t-b 3 w)$ pour cette raison. C'est aussi la tombe ( $\left.{ }^{c} b 3\right)$ d'Osiris, car c'est là que furent réunis ses membres, au côté de son père comme seigneur de l'occident». L'autre texte est gravé au début de la même colonne, il s'agit d'une description du temple nord à Pr-ntrr (Esna III, 196, $2)^{51}$ : «Pr-ntr du dieu des dieux, tombe ( $\left.{ }^{c} b 3\right)$ de Kematef, maison de Tanen, cité de Neith, place secrète des dieux antérieurs - les grandes paroles $(\underline{d}$ jisw) de la première génération - palais de Rê, château des $B a(h w t-b 3 w)$ de Chou et Tefnout, c'est l'enfer mystérieux que nul homme ne saurait voir, l'antre (imht) des dieux morts». - À la lecture de ces deux textes qui mentionnent les sept propos ( $\left.d_{3} i s w\right)$ de Méthyer à 'b3, on comprend mieux pourquoi l'évocation de la tombe d'Osiris a été placée au verset (7).

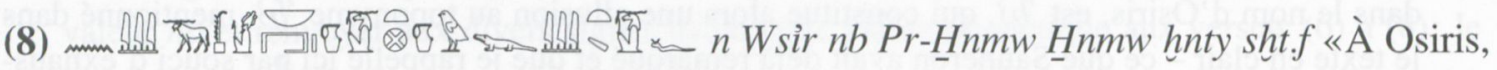
seigneur de $\operatorname{Pr}-\underline{H}$ Hmw (un temple au nord d'Esna), Khnoum qui préside à sa campagne».

A. Catégorie graphique (1a): Sauneron a naturellement remarqué que le signe de la campagne (ALh) renvoyait au même signe employé dans l'écriture de hnty-sht.f.

A. Catégorie graphique (2a) : la même remarque peut être faite au sujet du bélier ( $\bar{\aleph}$ ) figurant dans le nom d'Osiris, qui évoque le dieu criocéphale Khnoum ( ( ment.

A. Catégorie graphique (2b) : le crocodile ( que l'on trouve dans le nom d'Osiris du verset (9).

48 La lecture mhty «nord» est une proposition de S. Sauneron, Esna V, p. 294, n. (x), qui reconnaît dans le bélier ailé (

49 Traduction dans Esna V, p. 351.

50 À propos des sept $\underset{d}{\text { Bisw }}$, on verra en dernier lieu D. Mendel, op. cit., p. 116-120.

51 Traduction dans Esna V, p. 319. 
B. Catégorie graphique/phonétique (1b) : l'allusion suivante est plus complexe à comprendre, c'est un jeu autour du hiéroglyphe $r n p($ ( ). À première vue, on ne relève rien. Toutefois, l'existence d'une relation entre le signe $r n p$ et le groupe $̌ t\}$ du verset (9) peut être suggérée. En réalité, il ne s'agit pas d'un simple renvoi d'un élément $\mathrm{A}$ vers un élément $\mathrm{B}$, mais d'un renvoi de $\mathrm{A}$ à $\mathrm{C}$, l'intermédiaire $\mathrm{B}$ étant manquant. L'élément commun entre $\mathrm{A}$ et $\mathrm{C}$ est naturellement le vautour ( $\mathrm{A}$ ), dont on trouve de nombreux exemples dans les invocations à la bonne année, par exemple dans celles que $\mathrm{Ph}$. Germond a publiées dans la série Aegyptiaca Helvetica ${ }^{52}$ : le vautour ayant souvent la valeur št 3 , il peut servir de charnière entre les éléments A et $\mathrm{C}$.

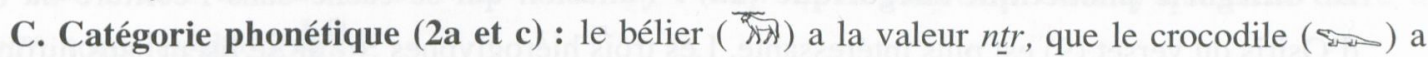
également dans le texte en clair et que l'on retrouve avec le signe $n \underline{t} r$ ( 7 ) du verset (10).

E. Catégorie allégorique (1a) : comme allusions allégoriques, on peut mentionner les signes de la campagne ( Khnoum, le seigneur de la campagne, une deuxième forme du dieu vénérée à Esna. Khnoum, le seigneur de la campagne, se manifeste sous la forme d'un crocodile ${ }^{53}$; son sanctuaire se trouve à $\mathrm{Pr}-\underline{H} n m w^{54}$.

E. Catégorie allégorique (1b) : une autre allusion allégorique se trouve encore dans l'emploi du signe de la campagne ( Rst-hwwt.s ( Plus bas, j'évoque, à l'occasion du commentaire du verset (9), des passages d'Esna (Esna III, 252, 28 (\$6) et IV, 425) qui indiquent que le lieu où les deux petites crocodiles étaient élevés se trouve précisément dans la campagne au nord d'Esna.

E. Catégorie allégorique (1b) : une dernière allusion relevant de la catégorie allégorique se trouve dans l'emploi du signe $r n p($ ( ) $)$, signifiant «rajeunir, être jeune». Celui-ci évoque ainsi les deux jeunes crocodiles aux mamelles de la déesse crocodilocéphale ( et les deux oisillons (2323) du verset (9).

F. Relation avec la litanie entière (1a) : la relation entre ce verset et la litanie entière est simple à établir : le nom du dieu se lit $\underline{H} n m w$, or, hnnw c'est aussi le chiffre huit. Il s'agit donc d'un calembour qui justifie la mention de Khnoum au verset $(8)^{55}$. On retrouve le même phénomène sur une tablette hiératique ${ }^{56}:$ au recto, qui contient des omina calendériques, le dieu du quatrième mois de la saison prt, c'est-à-dire du huitième mois, est précisément Khnoum. C'est le deuxième cas où le numéro d'un verset correspond à un des mois égyptiens (cf. supra verset [1]), on trouve un autre cas au verset (12).

52 Les invocations à la bonne année au temple d’Edfou (AegHelv 11), 1986, passim.

${ }^{53}$ Le célèbre hymne écrit avec une centaine des crocodiles est adressé à cette forme de Khnoum : Esna II, 126 (cf. Chr. Leitz, SAK 29 [2001], p. 251-276). L'utilisation d'un crocodile une allusion à ce fait - même s'il s'agit là d'une écriture assez courante.

54 Cf. S. Sauneron, Esna V, p. 53 et 316-317.

55 Pour cette sorte de jeu de mots, cf. Chr. Leitz, Tagewählerei, p. 276 avec n. 55.

56 P. Vernus, $R d E 33$ (1981), p. 92, texte p. 120, 1. 12. La traduction la plus récente se trouve chez H.-W. Fischer-Elfert, Altägyptische Zaubersprüche, 2005, 101-103. 
(9) mm of dans le Château des deux oisillons».

A. Catégorie graphique (1b) : le premier jeu graphique est la croix $(\&)$ que l'on retrouve dans le texte en clair $(\tilde{s} t 3)$, dans le nom d'Osiris du verset (10) et comme élément du signe whm (لٌ $(\mathfrak{l})$. A. Catégorie graphique (2b) : je ne suis pas convaincu de la réalité du deuxième jeu graphique : les deux oisillons ( 23 ) qui peuvent renvoyer au vanneau (10 du verset (10).

D. Catégorie phonétique/allégorique (1a) : l'allusion qui se cache dans l'écriture du nom d'Osiris du verset (9) est plus intéressante. Les trois hiéroglyphes of $R^{-}$se lisent sans difficulté wn s3 Rst-hwwt.s «la protection de la déesse Rst-ḥwwt.s existe». Or, plusieurs textes d'Esna évoquent non seulement la protection de cette déesse crocodilocéphale, mais aussi cette protection à $\underline{T} 3 w y$. Un premier exemple de ces textes se trouve entre les versets (61) et (63) de la litanie de Neith ${ }^{57}$ : «À Neith, maîtresse d'Esna, qui protège les deux oisillons de son fils, dans le District-des-deux-oisillons ( $33 w t \underline{t} 3 w y$ nw s3.s $m$ T $3 w y$ ) ; à Neith, maîtresse du Château-de-lamère (hwt-mwt), qui allaite ses deux fils crocodiles (rrt mshwy.sy); à Neith, qui veille sur ses châteaux (Rst-ḥwwt.s déterminé par qui tient embrassés les deux crocodiles dans ses bras $^{58}$ ». Le deuxième texte est extrait d'un hymne à Neith, déjà cité supra au verset (5). Le parallèle avec le premier texte est évident (Esna III, 252, 28 [§6]) : «Tu (Neith) es la maîtresse d'Esna, la protection de la campagne mystérieuse $\left(s 3 n^{59} s h t\right.$ št $\left.3 t\right)$ au nord des deux oisillons $(\underline{T} 3 w y)$, celle qui a allaité les deux crocodiles en leur nom de Chou et Tefnout, qui veille sur ses châteaux (c'est la déesse crocodilocéphale de notre verset), qui tient embrassés les deux crocodiles dans ses bras». La dernière inscription à livrer des informations sur ce sujet est gravée sous le règne de Ouespasianos (69-79) ${ }^{60}$ sur une des $\operatorname{architraves}^{61}$ : «Celle-ci, qui est sur son papyrus, brille à la campagne pure dans sa forme de déesse-vautour mystérieuse (ps $\underline{d}$ hryt-

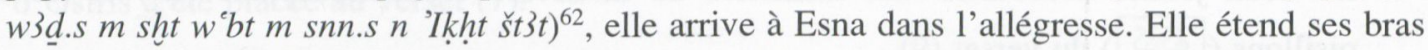
pour protéger le Château-des-deux-oisillons ( $h r n b i$ h hwt-ț $3 w y)$, pour protéger les dieux ( $h r m k$ $n t r w)$ dans le Château-de-Neith, elle contracte son aile comme protection (hr hwt), Rst-hwwt.s ${ }^{63}$ protège $(s 3 w . s)$ le vénérable pilier-Djed dans son palais».

E. Catégorie allégorique (1b) : le verbe $b s$ «entrer» est un renvoi à "k du verset (10), qui a plus ou moins le même sens.

${ }^{57}$ Esna III, 216, 13-14 (§61-63); traduction dans Esna VIII, p. 38.

58 hptt hh n Nt $m$ 'wy.sy, corriger Chr. Leitz (éd), LGG V, 126a-b, d'après D. Meeks, Mythes et légendes du Delta, p. 147, n. 513.

${ }^{59}$ Le $i b$ est probablement une faute pour le vase $n w$.

60 C'est-à-dire avant que ne soit décorée la colonne 3 qui porte la litanie d'Osiris et qui date du règne de Domitien (81-96).

${ }^{61}$ Esna IV, 425. Traduction chez A. von Lieven, Der Himmel über Esna. Eine Fallstudie zur Religiösen Astronomie in Ägypten am Beispiel der kosmologischen Decken- und Architravinschriften im Tempel von Esna (ÄgAbh 64), 2000, p. 104105.

${ }^{62}$ L'image de la déesse vautour mystérieuse ( pagnement. Dans ce contexte, on doit mentionner le fait que le texte Esna IV, 425 est situé sur l'architrave C, laquelle est précisément au-dessus de la colonne 3.

${ }^{63}$ Contra von Lieven, loc. cit. et ma propre interprétation dans $L G G$ IV, 720c ; je ne vois pas dans Rst-hwwt.s l'objet de la protection, mais - du fait de l'exemple d'Esna III, 252, 28 (§6) - le sujet, c'est-à-dire que Rst-ḩwwt.s se trouve en fonction topicalisée avant le verbe $s 3 w . s$. 
F. Relation avec la litanie entière (1a) : on peut aussi découvrir un élément qui permet d'établir une relation avec la litanie entière ou avec d'autres mots qui sont en mesure d'expliquer la position de ce verset. Les douze premiers versets peuvent être comparés aux douze heures du jour et les versets (13) à (24) aux douze heures de la nuit. La neuvième heure du jour s'appelle, à l'époque gréco-romaine, Dsrt-sšt 3 «celle à la forme secrète» ${ }^{64}$; l'allusion à la deuxième par-

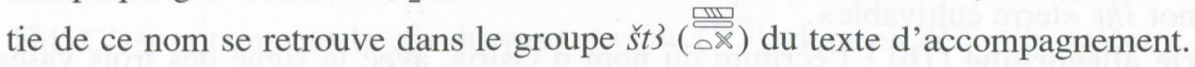

(10)

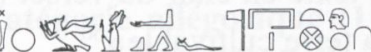
$n$ Wsir "k.f Pr-ntr tp sw $m \underline{d}$ « $\mathrm{A}$ Osiris, quand il entre à Pr-ntr, tous les dix jours».

A. Catégorie graphique (1a) : la première allusion graphique est évidente et elle a été naturellement relevée par Sauneron : le disque solaire (O) qui apparaît dans le nom d'Osiris et dans le texte en clair : 0 ก.

B. Catégorie graphique/phonétique (1b-c) : le signe de la jambe $(\stackrel{x}{)})$, avec sa valeur $w h m$, peut être admis comme une abréviation de $w h m^{-}{ }^{\circledR} h h$ «l'inondation». Il s'agirait donc d'un renvoi aux trois vases (200) du nom d'Osiris du verset (11), lesquels se lisent facilement $n w n$ «eau primordiale», mais aussi au dieu Hapy qui figure dans le nom d'Osiris du verset (12) ainsi qu'au groupe hipy «inondation» au verset (12).

E. Catégorie allégorique (1a) : ce même hiéroglyphe $w h$ m $\left(\mathbb{l}^{x}\right)$, qui signifie «répéter», renvoie également au groupe $t p s w m \underline{d}$, «au début de chaque décade», puisque quelque chose qui se déroule «au début de chaque décade», implique une répétition permanente.

F. Relation avec la litanie entière (1a) : le chiffre dix $(\cap)$ ne se trouve pas au verset $(10)$ par hasard.

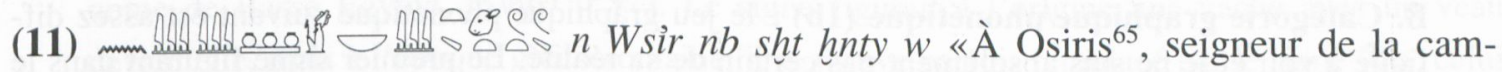
pagne, qui préside aux terres arables».

A. Catégorie graphique (1a) : les deux signes de la campagne (ALA ALA ), dans le nom d'Osiris, renvoient, bien entendu, au même signe dans le texte en clair.

A. Catégorie graphique (2b-c) : un autre jeu graphique se cache dans les trois vases $n w 000$ du nom d'Osiris qui renvoient aux trois autres vases à libation figurant au verset (12) ( $8 \mathcal{Z}^{\mathrm{Z}}$ ) et dans le texte en clair du verset (13) ( $(\zeta)$.

C. Catégorie phonétique (1b) : certains jeux phonétiques peuvent être bien plus raffinés et beaucoup plus difficiles à découvrir. Le deuxième vase figurant dans le nom d'Osiris au verset (12) a une lecture $s$, qui dérive du nom du vase swnw. Les deux hiéroglyphes de la campagne du verset (11) ont obtenu les valeurs phonétiques $w$ et $s$ par dérivation depuis les mots

64 Chr. Leitz (éd), LGG VII, 673b-c.

${ }^{65} \mathrm{~S}$. Sauneron, Esna VIII, 186, $\mathrm{N}^{\circ} 376$ ne fait pas de proposition pour justifier l'origine de la valeur phonétique $r$ du signe 000 . On peut éventuellement suggérer une dérivation depuis $r d \underline{d} w$ «écoulements», qui peut s'écrire avec les hiéroglyphes ૪૪૪: Edfou II, 38, 8 . 


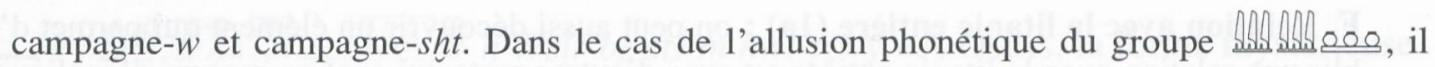
faut intervertir les deux : $s+w$. En combinaison avec le vase $n w$ du dernier signe, on obtient : $s+w+n w$, c'est-à-dire le nom swnw ( 80 ) du vase du verset (12).

E. Catégorie allégorique (1b) : les trois signes $s h$ «campagne» et le mot $w$ «terre arable» renvoient au mot $3 h t$ «terre cultivable».

E. Catégorie allégorique (1b) : l'écriture du nom d'Osiris, avec le signe des trois vases $n w$ ( $\cong 00)$, qu'on peut lire $n w n$, «eau primordiale», fait écho au texte en clair du verset (12): il $m$ $h^{i} p y$ iwh 3 ht «qui vient en forme d'inondation, qui inonde la terre cultivable» ${ }^{66}$.

E. Catégorie allégorique (1b-c) : l'épithète $n b$ sht «seigneur de la campagne» désigne une des deux formes principales de Khnoum à Esna et évoque ainsi les deux vases hnm ( $)$ ) qui entrent dans la composition du nom d'Osiris du verset (12) ainsi que dans le texte en clair du verset (13).

F. Relation avec la litanie entière (1a) : il m'a fallu un certain temps pour trouver la relation qui existe avec la litanie entière, c'est-à-dire une allusion au chiffre 11 . La solution se cache dans le hiéroglyphe de la campagne sht ( 19 h $)$. Chaque signe est, en réalité, composé de trois roseaux mais aussi de trois petits signes du terrain triangulaire $(\diamond)^{67}$. Trois signes de la campagne font ainsi neuf signes du terrain triangulaire auxquels on doit ajouter les deux autres $\diamond \mathrm{du}$ texte en clair. On obtient donc un total de onze signes du terrain triangulaire $(\diamond)$ au verset (11).

(12)

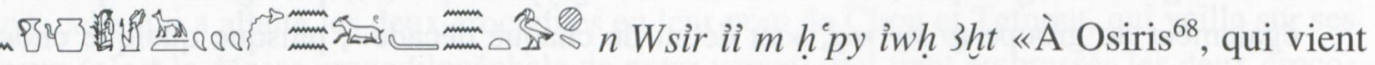
en forme d'inondation, qui inonde la terre cultivable».

A. Catégorie graphique (1b) : le vase $h n m(\mathcal{B})$ du nom d'Osiris renvoie au même vase figurant dans le texte en clair du verset (13).

B. Catégorie graphique/phonétique (1b) : le jeu graphique/phonétique suivant est assez difficile à voir et je ne suis absolument pas certain de sa réalité. Le premier signe figurant dans le

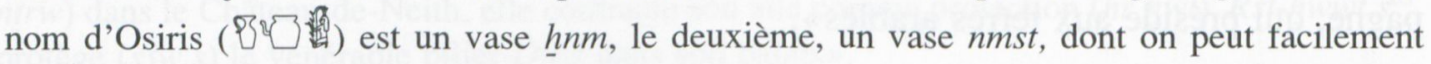
déduire une valeur $n m s$, le troisième est le dieu Hapy, qui se dissimule derrière un signe rnp. Il existe un mot $n t f$, «mouiller», et on peut conjecturer que celui-ci a pu être exprimé par ce signe mais, bien entendu, cela demeure conjectural. Dans ce cas, le nom d'Osiris du verset (12) aurait une deuxième lecture hnnm-snt.f «qui s'unit à sa sœur», allusion à l'épithète d'Osiris du verset (13). La curieuse faculté pour un signe plurilitère d'être employé pour noter des articulations appartenant à des mots différents existe à Esna, Sauneron en donne deux attestations avec le signe $n f r^{69}$.

${ }^{66}$ Cf. Esna VI, 494, 7-8 : «Osiris en tant que Noun, le grand qui inonde la terre $(i w h$ 3ht $)$ ».

${ }^{67}$ On verra les fac-similés chez D. Meeks, Les Architraves du temple d'Esna (Paléographie hiéroglyphique 1), 2004, p. 119,299 et 306.

${ }^{68}$ S. Sauneron, Esna VIII, p. 123, N ${ }^{\circ}$ 54, ne propose pas de dérivation pour la valeur phonétique $r$ de partant de la racine $r n p$, celle-ci doit être vraisemblable.

${ }^{69}$ Pour le principe général - l'utilisation d'un signe plurilitère pour noter des articulations appartenant à des mots différents - voir les remarques de S. Sauneron, Quatre campagnes à Esna (Esna I), 1959, p. 48-49. 
C. Catégorie phonétique (1a) : la divinité qui se cache derrière le signe $r n p$ (秒) n'est autre que le dieu de l'inondation, Hapy, qui renvoie donc au mot $h^{e} p y(000\}$

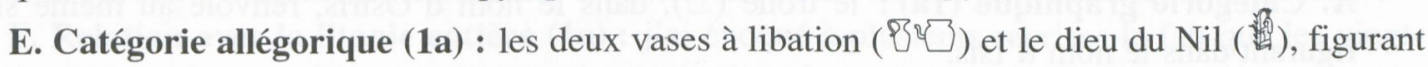
dans le nom d'Osiris, font allusion à la thématique générale du texte en clair : l'inondation du Nil. C'est notamment le cas du vase $s(w) n w\left({ }^{2} Z\right.$ ), dont on déduit la valeur $s$, qui était considéré comme le récipient contenant les écoulements $(r \underline{d} w)^{70}$, dont l'inondation tire son origine. E. Catégorie allégorique (1b) : l'allusion suivante est à rechercher dans l'épithète d'Osiris iwh 3 h , «qui inonde la terre cultivable». Cette expression évoque Osiris qui s'unit à sa sœur Isis, dont la meilleure illustration se trouve au chapitre XXXVIII du De Iside et Osiride de Plutarque : «De même qu'ils considèrent le Nil comme l'écoulement d'Osiris, de même ils déclarent et tiennent que la terre est le corps d'Isis, et non pas cependant toute la terre, mais seulement la partie que le Nil couvre et féconde en la pénétrant. Cet accouplement donne naissance à Horus. ${ }^{71}$

F. Relation avec la litanie entière (1a) : le verset (12) est un des rares cas où je suis en mesure de trouver trois motifs pour justifier sa position dans la litanie entière. Le premier est l'analogie que l'on peut établir entre les douze premiers versets et les douze mois du calendrier égyptien ; on a déjà vu ce cas au verset (8), la mention du dieu Khnoum renvoyait au chiffre huit et à Khnoum comme dieu protecteur du huitième mois. Le verset (12) est donc comparable au douzième mois, c'est le quatrième mois de la saison $̌ m w$. C'est pendant ce mois qu'avait lieu le solstice d'été et l'arrivée de l'inondation du Nil, c'est-à-dire le sujet même du verset. Une bonne référence à la date précise de ce phénomène naturel se trouve chez Hérodote, au livre II, chapitre $19^{72}$.

F. Relation avec la litanie entière (1a) : une autre allusion se trouve dans le hiéroglyphe de la jeune antilope bubale ( nome de Basse Égypte, $\underline{t} b$-ntrt ( Le signe figurait à l'origine une vache avec un veau

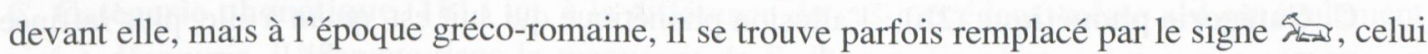
de la jeune antilope bubale.

F. Relation avec la litanie entière (1a) : la dernière allusion fonctionne avec l'homme qui se cache derrière le signe de l'année (䠳). H3p est un des mots signifiant «se cacher»; celui-ci peut s'écrire avec le signe de l'homme caché ( $\left.{ }^{2}\right)^{73}$. Or, il faut noter que le nom de la douzième heure du jour est justement $h 3 p t-\underline{d} s r t$, «Celle qui cache le secret» ${ }^{74}$; on en déduit donc un autre renvoi aux heures du jour et de la nuit.

70 Cf. J. Kettel, dans C. Berger et al. (éd.), Hommages à Jean Leclant (BdE 106, 3), 1994, p. 320-321, n. 46. Édité au même moment, le commentaire de H. Willems, The Coffin of Heqata (OLA 70), 1996, p. 107, apporte de nombreuses précisions. H. Willems fait remarquer que le vase snw sert aussi de déterminatif au verbe swnw «souffrir», lequel a aussi le sens de «se décomposer, se putréfier» (le locus classicus est Pyr. 617a). L. Pantalacci, GM 52 (1981), p. 60 suggère - d'après une remarque de G. Jéquier - le sens de «s'écouler».

${ }^{71}$ D'autres références sur le sujet chez Chr. Leitz, Tagewählerei, p. 70 ; au sujet du thème Osiris = l'eau de l'inondation et Isis = la terre. Cf. également, Chr. Leitz (éd), LGG VIII, 17b - 18a et 160b - 161a.

72 Cf. Chr. Leitz, Tagewählerei, p. 406.

${ }^{73} \mathrm{~Wb}$ III, 30.

74 Chr. Leitz (éd), $L G G \mathrm{~V}, 26 \mathrm{a}$. 
(13) mmn ภО

A. Catégorie graphique (1a) : le trône (』), dans le nom d'Osiris, renvoie au même signe figurant dans le nom d'Isis.

A. Catégorie graphique (2a) : un deuxième jeu graphique se trouve dans le disque solaire $(O)$ qui rappelle celui situé entre les deux cornes sur la tête d'Isis (

A. Catégorie graphique (2b) : le troisième jeu graphique fonctionne avec le sceptre en forme de papyrus que tient la déesse Isis ( $\left(\begin{array}{l}0 \\ 8\end{array}\right)$ qui évoque, naturellement, le signe du papyrus $(8)$ figurant dans le nom d'Osiris au verset (14).

C. Catégorie phonétique $(\mathbf{2 b})$ : la dernière allusion dans ce verset se cache dans le signe du siège, qui entre dans la composition du nom d'Isis, et se lit 3st, st. Il renvoie ainsi à la pustule $\left(8^{\circ} \mathrm{O}\right)$, présente dans le nom d'Osiris au verset (14), qu'on peut lire sty.

D. Catégorie phonétique/allégorique (1a) : on pourrait lire le groupe $\int \bigcirc, h t m p s d t$, «qui complète l'Ennéade», une allusion à Horus ${ }^{75}$, produit de l'unification d'Osiris avec sa sœur Isis.

E. Catégorie allégorique (1c) : hnnm, «s'unir (avec sens sexuel)», renvoie à wtt , «engendrer», qui figure dans le nom d'Osiris du verset $(15)^{76}$.

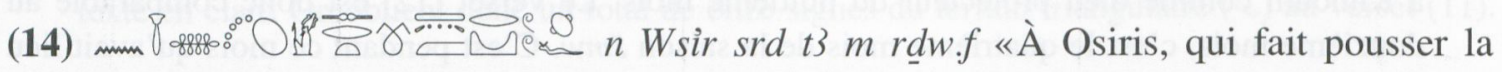
terre au moyen de ses sécrétions ${ }^{77}$.

A. Catégorie graphique (2a) : il n'y a qu'un seul jeu graphique ici, entre la pustule $\left(8^{0^{\circ}} \bigcirc\right)$ dans le nom d'Osiris et celle (ळ) dans le texte en clair.

C. Catégorie phonétique (1a) : la pustule $\left(8^{0^{\circ}} \bigcirc\right)$ est aussi à l'origine d'un jeu phonétique car sa valeur $r \underline{d} w, r d$ renvoie au verbe causatif $s r d$, «faire pousser».

C. Catégorie phonétique (2b) : l'allusion phonétique qui suit est, quant à elle, plus raffinée : le

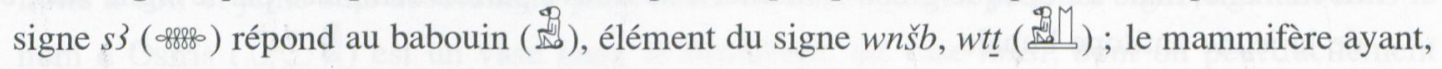
lui aussi, la valeur $s 3$, «protection».

C. Catégorie phonétique (2c) : l'allusion phonétique que l'on obtient à l'aide du signe du papyrus $(8)$, dans le nom d'Osiris, est encore plus sportive. Ce hiéroglyphe a la valeur $m h w$ «Basse Égypte», lecture également attestée pour le nid avec ses trois oiseaux (2989) au verset (16) et que l'on rencontre souvent à l'époque gréco-romaine pour écrire le mot mhyt «vent du nord».

F. Relation avec la litanie entière (1a) : pour la relation avec la litanie entière, on peut évoquer les inscriptions géographiques figurant aux soubassements de plusieurs temples ${ }^{78}$. On

75 Cf. l'épithète Htm-psdt-m-ntr-md «Celui qui complète l'Ennéade en tant que dixième dieu» comme désignation d'Horus et la littérature afférente dans Chr. Leitz (éd), $L G G$ V, 589a. - Je remercie M. Stefan Baumann pour cette suggestion.

76 Référence que je dois à $\mathrm{M}^{\text {me }}$ Victoria Altmann.

77 Le même ordre («inonder» au verset 1, puis «humidifier la terre» au verset 2 et enfin «laisser pousser les plantes» au verset 3) se retrouve également en Esna III, 225 (69-71), dans la litanie de Khnoum (voir aussi Esna III, 232 [110-111]).

78 Cf. H. Beinlich, Die «Osirisreliquien», p. 227. 
trouve par exemple mention des $r \underline{d} w$ dans la fameuse liste des nomes, située à l'extérieur du sanctuaire d'Edfou ${ }^{79}$ - bien entendu dans la légende du quatorzième nome de Haute Égypte et un $h w t-r \underline{d} w$ dans la même province est mentionné dans les papyrus hiératiques de Florence ${ }^{80}$. F. Relation avec la litanie entière (1a) : j'ai évoqué à plusieurs reprises le fait que les vingtquatre premiers versets pouvaient correspondre aux douze heures du jour et aux douze heures de la nuit. Le nom de la deuxième heure de la nuit, qui correspondrait au verset (14), est $S^{e} r t$ $n-n b . s$, «celle qui élève vers son seigneur», ou $S^{e} r t-n b . s$, «Celle qui élève son maître» ${ }^{81}$. Il est tout à fait possible que l'élément srt $(\vec{\nabla})$ dans ce verset renvoie à cette heure; la différence d'écriture est minime, d'autant plus que la consonne doit être supprimée, le e étant une consonne faible.

(15)

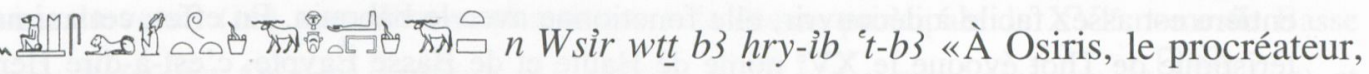
le $B a$ qui réside dans la demeure du $B a »$.

A. Catégorie graphique (2c) : la première allusion consiste en un jeu graphique avec les signes du lion : le lion ( $\Sigma 0$ ) du nom d'Osiris renvoie aux deux lits léontiformes ( verset (17).

B. Catégorie graphique/phonétique (1b) : la deuxième allusion n'est pas absolument sûre. On peut voir dans le groupe $t(\bar{\square}(-\vec{\square})$ du texte en clair au verset (15), un renvoi au $\square$ du nom d'Osiris du verset (16). En effet, le bol n'a pas seulement la valeur wsh, mais également, régulièrement, la valeur ${ }^{e}, \mathrm{c}^{\prime}$ est-à-dire que l'on peut lire ce signe ' $t$, en considérant que le $t$ du féminin ne s'écrit plus.

C. Catégorie phonétique (1a) : le babouin devant le signe $h n$ ( ( $w n s ̌ b$ ou $w t \underline{t}$ - renvoie naturellement au wț du texte en clair ; il a été, comme la plupart des renvois concernant ce verset, relevé par Sauneron.

C. Catégorie phonétique (1b) : ici, à la différence du cas précédent, même s'il est également aisé à découvrir, il manque dans le manuscrit de Sauneron. En effet, celui-ci n'a pas pris en

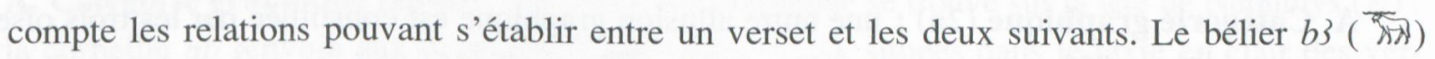
écrit à deux reprises dans le texte en clair du verset (15) renvoie phonétiquement aux trois oiseaux $b 3 w$ du groupe $h w t-b 3 w$ (16).

C. Catégorie phonétique (2a): le lion couché (\$D) peut être lu $n b$ comme le bélier ( Étant donné que la valeur $n b$ appartient, comme la lecture $n t r$, à de très nombreux signes, je ne suis pas absolument convaincu de l'intentionnalité réelle de ce renvoi.

C. Catégorie phonétique (2a) : le groupe constitué de la pièce d'étoffe et du lion couché ( $\bigcap_{\Xi \infty}$ ) peut se lire $s r$. Or, $s r$ est, par ailleurs, une désignation en égyptien du bélier : on obtien-

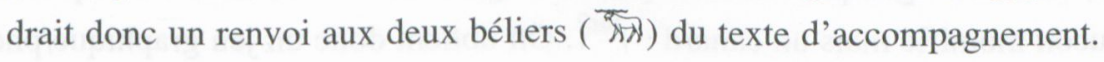

\footnotetext{
${ }^{79}$ Edfou I, 341, 5.

80 J. Osing, Gl. Rosati, Papiri geroglifici e ieratici da Tebtynis, 1998, p. 150-152.

${ }^{81}$ Chr. Leitz (éd), LGG VI, 193c - 194a.
} 
E. Catégorie allégorique (1b) : la théologie propre à Esna nous permet de relever un renvoi phonétique/allégorique pour ${ }^{e} t-b 3$ «la demeure du $B a »$. De fait, cette localité est connue par un passage de la grande litanie de Khnoum, située sur la colonne 5. On y lit ${ }^{82}$ : «À Khnoum, le grand bélier, qui réside dans la demeure du $B a$ (hry-ib 't b3), qui donne le souffle d'air aux dieux ( $r d i$ i $i s-n 3 w n$ ntrw)», le cobra dressé est à lire $n$. Ce qui est particulièrement notable ici, c'est la relation que l'on peut établir entre la ' $t$ - $b 3$ «la demeure du $B a$ » et le vent : de fait, on relève ainsi un renvoi au signe du nid avec les trois canards ( car, d'une part, la lecture mhyt «vent du nord» est bien établie pour ce signe et que, d'autre part, le créateur du vent est justement - selon la litanie de Khnoum -, le grand bélier qui réside dans la demeure du $B a$.

F. Relation avec la litanie entière (1a) : la relation qui s'établit entre ce verset et la litanie entière est assez facile à découvrir, elle fonctionne avec le babouin. En effet, cet animal caractéristique de Thot évoque le XV nome de Haute et de Basse Égypte, c'est-à-dire Hermopolis magna et parva, localités dont il était la divinité principale. C'est un des exemples les plus clairs relevant de cette catégorie.

F. Relation avec la litanie entière (1a) : une autre éventualité est de considérer que le babouin (용) symbolise la pleine lune, donc de supposer qu'il existe une possible équivalence entre les trente premiers versets de la litanie et les trente jours du mois lunaire. Dans ce cas, ce verset correspondrait au quinzième jour d'un mois lunaire, un moment où l'on peut observer la pleine

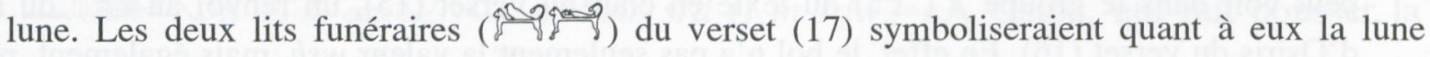
décroissante.

(16) mим des- $B a »$.

A. Catégorie graphique (2a) : le premier jeu graphique est déjà mentionné dans Esna VIII, le premier signe ( $\square$ ) du nom d'Osiris renvoie au $h w t-b 3 w\left({ }^{\square}\right.$ ) du texte en clair.

A. Catégorie graphique (2a) : une autre allusion graphique est constituée par les trois oiseaux dans leur nid ( Jabirous ( $)$ du texte en clair ; le nid lui-même, évoque la corbeille ( $\checkmark$ ) qui se trouve sur la tête de Nephthys (织) au verset (17) et celles des versets (17) et (18).

B. Catégorie graphique/phonétique (1b) : le jeu suivant, entre le nid avec ses trois oiseaux (30) et le lit avec un faucon momifié ( ? nid sont habituellement des canards et le signe du lit avec un faucon a normalement la valeur $s \underline{d} r$ «dormir». Or, un autre signe peut se lire $s \underline{d} r$, c'est également un lit, mais sur lequel ne se couche pas un faucon momifié mais un canard : On obtient donc un jeu graphique/phonétique entre les trois canards dans le nid et la valeur $s \underline{d} r$ du lit.

F. Relation avec la litanie entière (1a) : venons-en à la dernière catégorie, il s'agit de déterminer pourquoi ce verset se trouve en $16^{\mathrm{e}}$ position dans l'ensemble. La solution est à chercher

${ }^{82}$ Esna III, 232 (102). 
dans le toponyme $h w t-b 3 w$, «le château des $B a »$. Il s'agit tout à la fois d'un lieu situé à proximité d'Esna, mais également d'un sanctuaire localisé dans le $\mathrm{XVI}^{\mathrm{e}}$ nome de Basse Égypte $^{83}$. On peut aussi déterminer une autre relation entre le chiffre 16 et ce verset : dans les mythologies propres au XVI ${ }^{\mathrm{e}}$ nome de Basse Égypte. Une partie du signe $\square$ se lit $h w t$, «Château» et, étant donné que le signe des trois canards dans leur nid peut se lire mhyt,

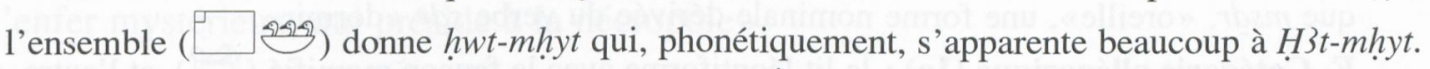
Hatmehyt était la déesse du XVI ${ }^{\mathrm{e}}$ nome de Basse Égypte, figurée sous la forme d'un dauphin ou d'un poisson beaucoup plus petit, le Schilbé, comme l'a récemment fait remarquer Jean Yoyotte ${ }^{84}$. L'identification du poisson itn étant pour l'instant peu significative pour notre propos, on retiendra que l'on peut reconnaître, dans les deux premiers signes figurant dans le nom d'Osiris du verset (16), une allusion phonétique à la déesse principale du XVI nome de Basse

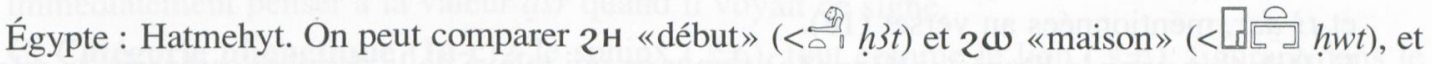

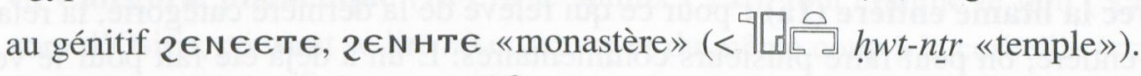

G. Autres allusions : le verset (16) est un des rares cas où l'on peut ajouter une observation qui n'entre précisément dans aucune des catégories que j'ai définies. Les trois canards dans leur nid (\$93) peuvent se lire mhyt, «vent du nord». Le deuxième groupe (fœ ) peut aussi valoir 'nht, «l'occident», tandis qu'au verset (17), le sarcophage $n b$ - $n h$ est écrit $\square$ $\leadsto$. Le troisième groupe, au verset (18), est $t 3$ - $\underline{d} s r$ «la nécropole», généralement située à l'ouest. Le dernier groupe du verset (19) est $M 3 n w$ «les montagnes de l'Occident». Ces quatre groupes, avec leurs mentions du nord et de l'ouest, sont gravées aux lignes 22 et 23 de la colonne 2 ; or, ces deux colonnes sont plus ou moins orientées vers le nord-ouest ${ }^{85}$.

(17) mm ngmon vie, qui préside au sarcophage».

A. Catégorie graphique (2a-b) : le premier jeu graphique se trouve sur la tête de Nephthys (婘) : la corbeille $n b$ renvoie aux deux autres corbeilles $(\smile)$, situées dans le texte en clair des versets (17) et (18).

A. Catégorie graphique (2b) : les deux lits léontiformes ( sition du nom d'Osiris au verset (17) renvoient au sphinx léontocéphale (@S) et au lion couché (\$D) figurant dans le nom d'Osiris au verset (18).

C. Catégorie phonétique (1a-b) : la déesse Isis (愖) peut avoir la valeur $n b$. On obtient ainsi un renvoi au cobra dressé $($ Cr) et à la corbeille $(\circlearrowleft)$ du même verset ainsi qu’à celle du verset

83 Gauthier, DG IV, 64.

84 J. Yoyotte, P. Vernus, Bestiaire des Pharaons, 2005, p. 242.

85 Voir le schéma qui accompagne l'édition du texte (Esna III, p. 56).

${ }^{86}$ L'origine de la valeur phonétique $w$ de ${ }^{\circledR}$ n'est pas bien établie. S. Sauneron, Esna VIII, p. 170, N ${ }^{\circ} 283$, suggère une dérivation depuis $w r s ̌$ «passer la nuit», mais il doit s'agir d’une confusion avec sd $r$, parce que $w r s ̌$ «passer le jour» n’est jamais déterminé ainsi. 
(18). Par ailleurs, il faut relever que le sphinx (S) et le lion couché (\$D) peuvent avoir la valeur $n b$.

C. Catégorie phonétique (2c) : un autre jeu phonétique peut aussi être noté: les deux lits léontiformes ( l'oreille de boeuf $\$$, introduite dans le nom d'Osiris du verset (19), qui peut se lire tant $s \underline{d m}$ que $m s \underline{d} r$, «oreille», une forme nominale dérivée du verbe $s \underline{d} r$ «dormir».

E. Catégorie allégorique (1a) : le lit léontiforme avec le faucon momifié (\$? ), et l'autre, avec la momie anthropoforme ( sarcophage $n b-{ }^{e} n h$ du texte en clair; la thématique funéraire est évidente.

E. Catégorie allégorique (1b-c) : par ailleurs, le sarcophage $n b-{ }^{i} n h$ du texte d'accompagnement du verset (17) fait allusion aux deux désignations de l'enfer et de la nécropole, la $d w 3 t s ̌ 3 t$ et $t 3$ - $\underline{d} s r$, mentionnées au verset (18).

F. Relation avec la litanie entière (1a) : pour ce qui relève de la dernière catégorie, la relation avec la litanie entière, on peut faire plusieurs commentaires. L'un a déjà été fait pour le verset (15) où j'ai proposé de voir dans le babouin une allusion au dieu lunaire Thot, en tant que pleine lune, au quinzième jour du mois. Si cette hypothèse est correcte, les deux lits funéraires du verset (17) constitueraient donc une symbolique de la lune décroissante ${ }^{87}$. L'autre commentaire concerne aussi la mort d'Osiris, puisque le verset (17) suggère la date du $17^{\mathrm{e}}$ Hathor, c'est-à-dire le $\mathrm{III}^{\mathrm{e}}$ mois de la saison $3 h t$, le jour 17. Les théologiens égyptiens, suivant une très longue tradition, ont situé à cette date le meurtre d'Osiris. La plus ancienne mention de cette précision se trouve en effet dans les calendriers des jours fastes et néfastes. Fr. Chabas releva le premier, dans son édition du papyrus Sallier IV, publiée en 1869, la relation que l'on pouvait établir entre ce texte et certains passages de Plutarque ${ }^{88}$. Plutarque étant plus ou moins contemporain de la décoration des colonnes d'Esna, savoir si l'auteur de la litanie d'Esna a trouvé cette information dans un texte grec ou plus vraisemblablement dans un texte égyptien n'a, en réalité, aucune importance : la tradition égyptienne fixant la mort du dieu à cette date existait sans doute déjà dès le Nouvel Empire ${ }^{89}$.

F. Relation avec la litanie entière (1a) : une autre relation avec la litanie entière peut être suggérée ; j'ai en effet, à plusieurs reprises, fait remarquer que les vingt-quatre premiers versets pouvaient correspondre aux douze heures du jour et aux douze heures de la nuit. Ainsi, le verset (17) correspondrait à la cinquième heure de la nuit. Le nom de cette heure est, à l'époque

87 Cf. R. A. Parker, The Calendars of Ancient Egypt (SAOC 26), 1950, p. 11-14. Le 17 jour est le cas le plus fréquent, mais tous les jours entre le $16^{\mathrm{e}}$ et le $19^{\mathrm{e}}$ sont possibles; on verra les calculs de H. Mucke chez Chr. Leitz, BSEG 18 (1994), p. 54-58.

${ }^{88}$ Chr. Leitz, Tagewählerei, p. 127-129 avec les citations de Plutarque (chapitres 13 et 39 ) ; Fr.-R. Herbin, BIFAO 88 (1988), p. 99-100, 103.

${ }^{89}$ S. Sauneron précise que la décoration de la colonne 3 fut réalisée sous Domitien (81-96), la poursuite du texte sur la colonne 2, daterait du règne de Trajan (98-117). Le cartouche assigné par Sauneron à Domitien (e. g. Esna VIII, p. 40) est encore lu Trajan par J. von Beckerath, Handbuch der ägyptischen Königsnamen (MÄS 49), 1999, p. 259 (il ignore probablement les remarques de S. Sauneron, BIFAO 53 [1953], p. 4952). Plutarque vécu environ de 45 à 120 de notre ère ; son livre, De Iside et Osiride a été composé quelques années avant 115 (selon H. Görgemanns, Plutarch, Drei religionsphilosophische Schriften, 2003, p. 340). La date était également connue à Dendara sous les derniers Ptolémées, cf. Chr. Leitz, Die Außenwand des Sanktuars in Dendara (MÄS 50), 2001 , p. 333. 
gréco-romaine, $n b t^{-}{ }^{2} n h$ " «la maîtresse de la vie» ${ }^{90}$ et les deux épithètes d'Osiris - $n b$ ' $n h$ «seigneur de la vie» et hnty $n b-{ }^{-} n h$ «qui préside au sarcophage» - font allusion au nom de la cinquième heure de la nuit $n b t$ - $^{e} n h$ «la maîtresse de la vie».

(18)

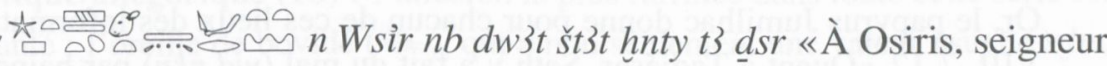
de l'enfer mystérieux, qui préside à la nécropole».

B. Catégorie graphique/phonétique (1a) : le lion couché (\$D), entrant dans la composition du nom d'Osiris, renvoie probablement au groupe $d s r$ (S) du texte en clair. En effet, on trouve couramment à l'époque gréco-romaine le lion couché, tenant entre ses pattes un couteau (\$S), avec cette valeur phonétique. Ici, bien sûr, le couteau est absent, mais chaque prêtre pouvait immédiatement penser à la valeur $\underline{d} s r$ quand il voyait ce signe.

C. Catégorie phonétique (1a-c) : le sphinx (\$S), tout comme le lion (\$D), figurant dans le nom d'Osiris, peuvent se lire $n b$ et renvoyer du même coup à la corbeille $(\circlearrowleft)$ du texte en clair et au cobra dressé ( $(\hat{T})$ des versets (19) et (20), qui ont la même valeur phonétique.

C. Catégorie phonétique (2a) : le jeu phonétique suivant n'est pas bien établi. En effet, le premier signe apparaissant dans le nom d'Osiris (a S0) est inconnu. Il a une certaine ressemblance avec le signe $ी$, utilisé généralement comme déterminatif mais rarement comme idéogramme pour $t m 3$ «la natte». Si ce rapprochement est avéré, alors, on peut considérer que le premier signe est une dentale et dès lors, avec le $s$ dérivé du sphinx $s \check{p}$ et le $r$ issu de la lecture $r w$ du lion, une lecture $\underline{d} s r$ est envisageable, celle-ci constituerait donc un renvoi au groupe du texte en clair.

C. Catégorie phonétique (2b) : l'adjectif $\underline{d} s r$ (S), dans le texte d'accompagnement du verset (18), renvoie peut-être, par calembour, à la couronne rouge ( d'Osiris du verset (19), celle-ci pouvant aussi se lire $d \check{r} r t$ «la couronne rouge». Le nom de la

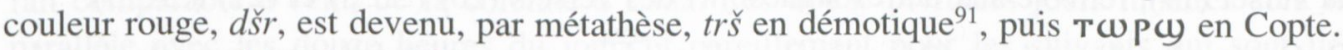

E. Catégorie allégorique (1b) : les allusions suivantes sont à rechercher dans les deux désignations du monde funéraire, la $d w 3 t$ št $3 t$ «l'enfer mystérieux» et $t 3-\underline{d} s r$, un mot désignant «la nécropole». Ces deux syntagmes renvoyant soit à imht, qui désigne aussi une partie du monde souterrain, soit à $M 3 n w$, la montagne occidentale.

F. Relation avec la litanie entière (1a) : la première relation qui peut s'établir avec la litanie entière n'est pas difficile à trouver. C'est naturellement le lion (\$D) ou les deux lions, sphinx inclus, qui renvoient tous les deux à la déesse Bastet, parfois lionne, parfois chatte, qui était la divinité principale de Boubastis, capitale du XVIII ${ }^{\mathrm{e}}$ nome de Basse Égypte ${ }^{92}$; ce qui justifie sa position au verset (18).

90 Chr. Leitz (éd.), $L G G$ IV, $28 \mathrm{a}$.

91 Erichsen, DemGl, p. 658 ( $t$ šr et $t r \check{s})$.

92 Il faut mentionner la fête du nome boubastide au $18^{\mathrm{e}}$ Payni (Edfou I, 335, 6) et l'allusion dans les calendriers des jours fastes et néfastes (Chr. Leitz, Tagewählerei, p. 361-363). 
F. Relation avec la litanie entière (1a) : l'autre relation avec la litanie entière se cache dans l'expression $t 3$ - $\underline{d} s r$ «la nécropole»; $t 3$ - $\underline{d} s r$ est en effet une localité située dans le XVIII ${ }^{\mathrm{e}}$ nome de Haute Égypte, attestée à plusieurs reprises dans le papyrus Jumilhac. On la trouve notamment mentionnée dans la liste de trente et une localités du XVIII ${ }^{\mathrm{e}}$ nome (pJumilhac VII, 14). Or, le papyrus Jumilhac donne pour chacun de ces lieux des informations précises : pJumilhac VIII, 7-12 «Quant à Tadjéser, Seth y a fait du mal ( $w d n k n)$ par haine d'Osiris : il dépeça tous les membres de son ennemi, mais Anubis, s'en étant allé pour les rechercher dans les villes et dans les nomes, revint avec eux dans le district de Dounâouy/Hardaï, et ils furent réunis dans la nébride ${ }^{93}$ et placés dans le pavillon divin en qualité de $\underline{d} s r w r$ (peut-être le grand inaccessible), de telle sorte qu'aucun dieu ne pût les voir. On l'appela Tadjéser à cause de cela». Il y a un autre petit passage qui évoque Anubis de $t 3-\underline{d} s r$ (pJumilhac IV, 18) : «Quant à Anubis, maître de la nécropole, qui est dans ce lieu, c'est Osiris quand il apparaît, au début de chaque décade, pour pourvoir ses tables d'offrandes». Tous ceci justifie l'évocation de $t 3$ - $\underline{d} s r$ au verset (18) de la litanie d'Osiris à Esna.

F. Relation avec la litanie entière (1a) : enfin, il y a une dernière allusion au chiffre 18 cachée dans notre verset et je dois admettre que la combinaison de toutes ces allusions dans ce verset est impressionnante. Comme au verset précédent, celle-ci découle de l'assimilation des vingtquatre premiers versets aux douze heures du jour et aux douze heures de la nuit : le verset (18) correspondrait ainsi à la sixième heure de la nuit. Celle-ci s'appelle $n b t \underline{d}$ srt št $3 t$ «la maîtresse des lieux inaccessibles et mystérieux ${ }^{94}$; tous les éléments de son nom se retrouvent dans les deux épithètes d'Osiris (nbt $d w 3 t$ št $3 t$ hnnty $t 3 \underline{d} \boldsymbol{d} \boldsymbol{r}$ ) du verset (18).

(19) mm no d'imht (une autre désignation de l'enfer), qui préside à la montagne occidentale».

A. Catégorie graphique (2b-c) : le signe $h w t($ (द्व $)$ «château», figurant dans le nom d'Osiris au verset (19), renvoie aux autres signes ḥt des versets (20) (

C. Catégorie phonétique (2a) : l'œil oudjat ( $)$ peut se lire $b 3 k$, par exemple dans le nom $B 3 k t$, «l'Égypte» et renvoie ainsi à l'arbre $\}$, qui peut aussi se lire $b 3 k$.

C. Catégorie phonétique (2a) : la couronne rouge ( $/$ ) peut se lire $m h \mathrm{~h} w$, comme dans le nom désignant la Basse Égypte. On obtient donc un calembour avec imḥt, «au-delà», en ne considérant que les consonnes fortes.

C. Catégorie phonétique (2a-b) : l'œil oudjat ( $)$ a aussi la valeur $n \underline{n} r$, comme souvent dans le mot $n t r t$, «l'œil divin», et renvoie ainsi aux deux crocodiles (

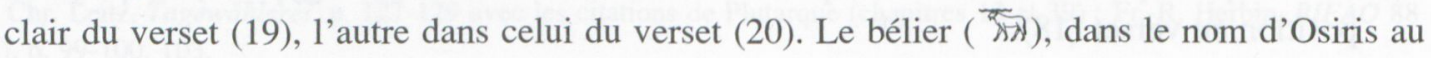
verset (20), peut aussi se lire $n \underline{t} r$.

${ }^{93}$ La mention de l'imiout peut aussi donner l'explication du signe $\_$, jusqu'à présent inconnue, avec sa valeur phonétique $w$ (cf. S. Sauneron, Esna VIII, p. 179, $\mathrm{N}^{\circ} 333$ ). Je suggère de voir ici un signe $₫$ mal fait avec sa valeur habituelle $w t(>w)$.

${ }^{94}$ Chr. Leitz (éd.), LGG IV, 172a-b. 
D. Catégorie phonétique/allégorique (1a) : on connaît aussi, pour l'œil oudjat ( $)$, une valeur wnmt, «l'œil droit», qui signifie également «occidental», il évoque ainsi M3nw, la «montagne occidentale».

D. Catégorie phonétique/allégorique (1b) : l'allusion la plus raffinée dans toute cette série est sans conteste la suivante : l'oreille de bovidé (\$), qui a non seulement une valeur usuelle $s d m$, mais aussi une lecture 'Iwnw' ${ }^{95}$. Or, 'Iwn, 'Iwnw, 'Iwnyt etc., ne renvoient pas seulement à Héliopolis, Dendara et Esna, mais également à Ermant. C'est précisément là que se trouvait le Bucheum, le sanctuaire du taureau Boukhis. Le nom égyptien de celui-ci était hwt-Tm (s). On obtient donc, à travers l'oreille de bovidé, une allusion discrète au toponyme $h w t$-Tm dans le texte en clair du verset (20).

E. Catégorie allégorique (1b) : l'épithète hnty $M 3 n w$, «qui préside à la montagne occidentale», est employée en Esna II, 106, 1 pour désigner Atoum ; cela constitue donc un rappel du hwt-Tm ( $)$ du texte en clair du verset (20).

F. Relation avec la litanie entière (1a) : nous arrivons maintenant à la dernière catégorie, il s'agit de déterminer si l'on peut relever dans ce verset un renvoi au chiffre 19. En fait, celui-ci se cache dans l'oreille de bovidé (両). Dans une inscription accompagnant une représentation d'Horus d'Edfou, plantant son pieu dans le dos d'un crocodile, on lit ${ }^{96}$ : «Paroles à dire par Horus d'Edfou, le grand dieu maître du ciel, le héros vigoureux avec son harpon, qui fait la protection dans la place d'Osiris, qui massacre ceux qui se rebellent contre lui, éternellement. Je poignarde ( $h n h h n)$ le Voleur ( $h w r r^{e}$, déterminatif : (jour de) Celui-qui-entend-ses-paroles, au moment de l'Uræus-qui-combat-pour-son-maître». L'expression $s \underline{d} m-m d w . f$ «celui qui entend ses paroles» est la désignation du dix-neuvième jour du mois lunaire ${ }^{97}$, d'où provient notre allusion avec l'oreille de bovidé du verset (19). Cela n'est probablement pas tout, la hryt-tp ‘ $h 3 t h r$ nb.s «l'Uræus qui combat pour son maître» est également le nom de la septième heure de la nuit ${ }^{98}$; on retrouve à Dendara, un système tout à fait comparable à celui de la colonne d'Esna. Les douze premiers jours lunaires sont mis en parallèle avec les douze heures du jour, et pareillement pour les suivants qui sont liés aux heures de la nuit. La position de la septième heure au dix-neuvième jour lunaire est donc justifiable. Il reste toutefois deux détails à préciser : la présence du crocodile dardé par le pieu du dieu, d'une part, et l'uræus figurant dans le nom de l'heure nocturne, d'autre part. Il y a non seulement une relation entre l'oreille de boeuf et le nom du dix-neuvième jour lunaire, mais aussi entre Seth sous la forme d'un saurien et le crocodile (sw) figurant dans le texte d'accompagnement (hnty M3nw) et enfin, entre l'Uræus en tant que nom de la septième heure de la nuit et le cobra (ל) ( $\mathrm{nb}$ imht) dans le texte en clair.

\footnotetext{
95 On peut expliquer cette graphie comme une écriture phonétique dérivée de la valeur idn, pour les détails voir S. Sauneron, Villes et légendes d'Égypte (BdE 90), 1983, p. 14-17.

${ }^{96}$ Dendara X, pl. 198, 1. 205-210.

$97 \mathrm{~Wb}$ IV, 388, 10.

${ }^{98}$ Dendara X, 363, 5-7 ; pour le nom de la septième heure de la nuit voir Chr. Leitz (éd.), LGG II, 396c - 397a et V, $447 \mathrm{a}-\mathrm{b}$.
} 
(20) mm place haute $(?)^{99}$, qui préside au Château-d'Atoum ${ }^{100} »$.

A. Catégorie graphique (2a) : le trône sur la tête d'Isis renvoie à celui qui figure dans le texte en clair.

C. Catégorie phonétique (1a-c) : la déesse $N b t-h w w t$, Nephthys (鹃) renvoie, phonétiquement, à une multitude de hiéroglyphes. On reconnaît la deuxième partie de son nom ( $h w t)$ dans les deux groupes $h w t-T m$ ( sa valeur $n b$, fait référence aux deux cobras ( $($ R) des versets (20) et (21) qui ont la même valeur phonétique, au crocodile ( se lisent $n b$ et naturellement au signe de la corbeille $(\circlearrowleft)$ du verset $(22)$.

C. Catégorie phonétique (2a) : la déesse Isis (陆) a parfois la valeur $p t$, comme le signe du ciel $(\longmapsto)$ dans le texte d'accompagnement.

C. Catégorie phonétique (2a-c) : le bélier ( ture du nom d'Osiris au verset (20), se lisent $n b$ et renvoient aux deux cobras ( ( R) des versets (20) et (21), au crocodile ( (22) et au signe de la corbeille $(\circlearrowleft)$ du verset $(22)$. En le lisant $n \underline{t} r$, le bélier peut aussi, encore une fois, rappeler le crocodile (एक्ष) qui a la même valeur.

C. Catégorie phonétique (2b) : on peut lire le groupe du bélier et de la déesse Isis ( le $s$ dérivant de la lecture $s r$ du bélier et le $n b$ d'Isis. On obtient donc une allusion au signe de l'étoffe $(\bigcap)$, présent dans le nom d'Osiris du verset (21) ; il s'agit aussi d'une abréviation courante de $s n b$, «être en bonne santé».

F. Relation avec la litanie entière (1a) : la relation entre le chiffre 20 et le contenu du verset (20) n'est pas difficile à trouver : le dieu principal du XX $X^{\mathrm{e}}$ nome de Haute Égypte était précisé-

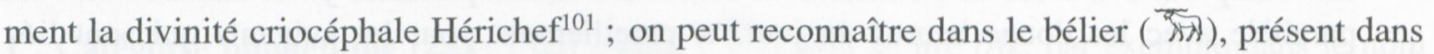
le nom d'Osiris, une représentation de ce dieu et un renvoi au chiffre 20.

(21) mm des-provendes, qui préside à la campagne».

C. Catégorie phonétique (1c) : la jambe $(\varangle)$ a pour valeur normale $r d$ d'où dérive le $r$ dans le nom d'Osiris; on voit donc un renvoi graphique à la plante $r d(\varnothing)$ figurant dans le nom d'Osiris du verset (23).

\footnotetext{
99 M. Daniel von Recklinghausen me renvoie à Esna II, 19, 2 et à D. Kurth, GM 19 (1976), p. 35-37 et il suggère de lire ici $n b$ 3st «seigneur du ciel».

100 Il n'y a pas ici d'indice qui permette de déterminer si ḩwt-Tm est une allusion au temple d'Atoum, au Bucheum ou à un toponyme du VIII ${ }^{\mathrm{e}}$ nome de Basse Égypte (Gauthier, DG IV, p. 53).

101 Le meilleur exposé demeure celui de Mohamed Gamal el-Din Mokhtar, Ihnâsya El-Medina (Herakleopolis Magna). Its Importance and its Role in Pharaonic History (BdE 40), 1983, p. 139-175.
} 
C. Catégorie phonétique (2a): pour ce qui relève de la catégorie phonétique, on peut mentionner le poussin de caille ( $\mathrm{f}$ ), dans le nom d'Osiris, avec sa valeur usuelle $w$ : ce hiéroglyphe renvoie ainsi au signe de la campagne ( $(20 h)$ ), qui a la même valeur phonétique.

C. Catégorie phonétique (2a) : une autre lecture possible pour le groupe $\bigcap \int$ est $s 3 \underline{t}^{102}$. On obtient ainsi une allusion aux deux hommes assis, tenant un vase à libation (\$2), qui peuvent également avoir la valeur $s 3 \underline{t}$.

D. Catégorie phonétique/allégorique (1a) : ce même groupe ( $\left(\int\right)$ peut, plus conventionnellement, se lire $s r d$, qu'on identifie aisément avec le verbe $s r d$, «faire pousser (spécialement la végétation)». Ceci constitue donc une allusion phonétique/allégorique au mot sht, «campagne» et au mot $\underline{d} f 3 w$, «nourriture/provende», c'est-à-dire les produits de la campagne.

E. Catégorie allégorique (1b-c) : la jambe d'Osiris $(\mathbb{S})$ est, selon beaucoup de traditions, la source de l'inondation, celle-ci s'écoule de la jambe gauche d'Osiris ${ }^{103}$. On obtient donc une allusion allégorique à la thématique de l'eau, laquelle est évoquée dans les deux versets suivants. F. Relation avec la litanie entière (1a) : il faut peut-être également reconnaitre une allusion au $\mathrm{XXI}^{\mathrm{e}}$ nome de Haute Égypte dans le nom d'Osiris ( 18 ). Jean Yoyotte a rassemblé quelques graphies du nom de la métropole de ce nome, $S m n w-H r$, et en a fait une classification ${ }^{104}$. Celuici peut s'écrire de nombreuses manières différentes et, dans de nombreux cas, le faucon $\mathrm{Hr}$ se trouve au début du nom: 100 m. $\int$ est $m n t$ «la cuisse», utilisée dans la litanie de Khnoum pour écrire $m$. On peut donc admettre une lecture smn pour les deux derniers signes, c'est-à-dire la première partie du toponyme. Toutefois, il manque le faucon ( on ne lit en effet qu'un ; or, normalement les deux signes ne se confondent jamais. Cependant, dans les litanies d'Esna, Sauneron a relevé des cas où le vaut pour c'est-à-dire qu'il a la valeur $h$, ou bien encore qu'un est employé pour un avec la valeur $w^{105}$. Par conséquent, je suggère de voir dans cette graphie d'Osiris une étrange allusion au nom de la capitale du XXI ${ }^{\mathrm{e}}$ nome de Haute Égypte : Smnw-Hr.

F. Relation avec la litanie entière (1a) : la jambe est aussi la clef pour comprendre la relation du verset (21) avec la litanie entière. Encore une fois, la solution est à rechercher dans les processions géographiques des nomes égyptiens. Dans la liste, citée à plusieurs reprises, située à l'extérieur du sanctuaire du temple d'Edfou, la notice relative au XXI ${ }^{\mathrm{e}}$ nome de Haute Égypte rappelle la tradition qui indique que c'était là que l'on conservait la jambe gauche d'Osiris ${ }^{106}$.

${ }^{102} \mathrm{La}$ lecture $3 t / 3 \underline{t}$ se retrouve dans le titre administratif $\int_{0}$, longtemps lu wirtw (e. g. Wb I, 288, 9, sq). La lecture 3tw/3tw a cependant été bien établie: G. Posener, RdE 15 (1963), p. 127-128; H. Wild, BIFAO 69 (1970), p. 117-121 (cf. J. Baines, dans J. Osing, G. Dreyer, Form und Mass. Festschrift für Gerhard Fecht [ÄAT 12], 1987, p. 47). Quelques références datant de l'Ancien Empire chez R. Hannig, Ägyptisches Wörterbuch I, 2003, p. 21-22.

103 Les sources égyptiennes à ce sujet sont nombreuses, cf. H. Beinlich, Die "Osirisreliquien», p. 209-213 ; voir aussi R. Merkelbach, ZÄS 99 (1973), p. 116-127.

104 Cf. J. Yoyotte, RdE 13 (1961), p. 80-92, et une sélection des graphies p. 82.

105 Cf. S. Sauneron, Esna VIII, p. $140, N^{\circ} 133(\Omega=w) ;$ p. $144, N^{\circ} 152(\Omega=h)$ et p. $130, N^{\circ} 86(\Omega=m$, dérivé de $m n t)$.

106 H. Beinlich, Die «Osirisreliquien», p. 236-238. 
(22) mm lac divin, à la tête du $S 3 w$ - hnnm (un autre lac au nord d'Esna)».

A. Catégorie graphique (2a-b) : l'eau qui s'écoule du vase des deux hommes (新), dans le nom d'Osiris, renvoie au hiéroglyphe de $m w(\underset{m m}{\sim m})$ du texte en clair des versets (22) et (23).

A. Catégorie graphique (2b) : le vase à libation que tient l'homme assis (5 vase (I) dans le nom d'Osiris du verset (23).

C. Catégorie phonétique (2a-b) : la possibilité que le lion couché (\$D), avec la valeur $n b$, puisse renvoyer aux deux corbeilles ( $\supset$ ) des versets (22) et (23) ainsi qu'au crocodile ( du verset (23), qui a la même lecture, est à la fois banale d'un certain point de vue et incertaine de l'autre.

E. Catégorie allégorique (1a-b) : les allusions allégoriques sont plus intéressantes. Il est évi-

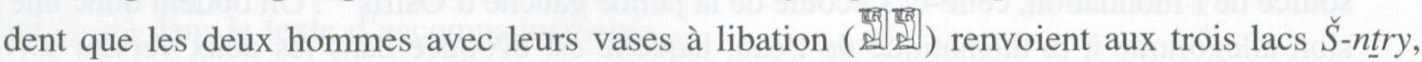
$S 3 w$-hnnm et $\breve{S}$-ďsr mentionnés aux versets (22) et (23). On ne peut rien dire du premier, le $\breve{S}$ ntry «le lac divin», puisqu'il n'est attesté qu'ici. En revanche, les deux autres sont mieux connus $^{107}$ : ces plans d'eau jouent un rôle pendant la fête des 19 et 20 Épiphi, c'est-à-dire les fêtes de prendre la houlette et de la victoire ${ }^{108}$. Dans le calendrier des fêtes, on relève, pour le 19 Épiphi, l'information suivante ${ }^{109}$ : «Apparition de Khnoum, le bon protecteur ( $p 3 n h w n f r$ ) à $\operatorname{Pr}-\underline{H} n m w^{110}$; faire halte à l'embouchure de l'Étang rouge $(\breve{S} \text { - } d \check{s} r)^{111}$, son beau visage étant tourné vers le lac de $S 3 w-\underline{h} n n^{112}$.» Selon un autre texte d'Esna ${ }^{113}$, des eaux, qui sont au nord du temple de Khnoum de la campagne, se trouvent entre Pr- $\underline{H} n m w$ et $\operatorname{Pr}$-ntr : : QQuant à ce Noun, on l'appelle $S 3 w$-hnnn. C'est l'endroit où se produisit le massacre $(s t g b g b)$.» Un autre passage précise $^{114}$ : "Quant à Khnoum le bon protecteur, souffle bienfaisant des dieux et des hommes, il est venu afin d'assurer la sauvegarde de Rê dans $S 3 w-\underline{h n n}$, et de maintenir sains et saufs les matelots de Chou à l'intérieur de ce lac.» Un chant du matin contient l'indication que Khnoum qui se trouve dans le lac de $S 3 w$ - $h n n$ est dans sa forme de crocodile sacro-saint $(m s h \underline{s} \text { šs })^{115}$. Mais, le texte qui nous intéresse avant tout ${ }^{116}$ se trouve au voisinage immédiat du célèbre hymne des crocodiles (Esna II, 126) ${ }^{117}$, il n'est malheureusement pas très bien conservé, notamment dans les trois premières colonnes : "Il (= Khnoum) [prit] sa [route] contre eux, chargé de [ses] armes de combat. Il... eux... lion ${ }^{118}$ (le premier signe marqué)... seul, il... à

107 Cf. S. Sauneron, Esna V, p. 325-326, 337-340 et 374-375.

108 hb ț 3 wn ha kn n Hnmw (Esna III, 259, 1).

109 Esna II, 77, 16 = Esna V, p. 26 et 312.

110 Voir S. Sauneron, Esna I, p. 28-29 (un sanctuaire situé au nord-ouest d'Esna, consacré à Khnoum-Rê, seigneur de la campagne).

111 Le passage qui correspond à la version la plus complète se trouve en Esna III, 197, 14 = Esna V, p. 335.

112 S. Sauneron (Esna V, p. 338, n. b) pense que $S 3 w-h n m / n$ est le nom du lac rouge.

113 Esna III, 196, 2-3 = Esna V, p. 324.

114 Esna III, 197, 14 = Esna V, p. 338.

115 Esna III, 259, 1-2 = Esna V, p. 356-357.

116 Esna II, 127 = Esna V, p. 374-375.

117 Voir le schéma dans Esna II, p. 238.

${ }^{118}$ La partie postérieure du hiéroglyphe du lion est conservée au début de la ligne 2. 
une multitude ; il fit un grand carnage (hryt ${ }^{~} 3 t$ ) parmi eux ; et ils churent dans l'eau sous lui». Le texte continue: «Il changea d'aspect et pris la forme d'un crocodile». Sauneron supposait que l'endroit où se déroulaient ces évènements était l'étang rouge - précision probablement due à la couleur du sang des ennemis. Il mentionne un texte selon lequel Khnoum avait pris, dans un premier temps, la forme d'un grand crocodile dans le Noun et que, par la suite, il se transforma en lion pour poursuivre ses ennemis ${ }^{119}$. En conclusion, on peut suggérer que, pour ce verset, ces mythes justifient la présence du lion (\$D) et du crocodile ( des deux lacs $S 3 w-\underline{h} n m$ et $\check{S}$-d $\check{s}^{120}$.

(23) ..m [ees r 19 de l'Étang rouge ${ }^{121}$, qui préside à la butte des plantes $k 3 k 3$ (une localité normalement située près de Hérakléopolis ${ }^{122}$ )».

A. Catégorie graphique (2c) : le disque solaire dans la barque (@OS), dans le nom d'Osiris au verset (23), renvoie au disque solaire, mais écrit avec le cobra ( $\bullet$ ), du verset (25).

B. Catégorie graphique/phonétique (1b) : la barque avec le disque solaire (@S) s'apparente beaucoup à la barque $w h^{\circ}(®)$. Cette dernière a aussi, par métathèse, la valeur $w^{c} h$ et elle renvoie ainsi au nom d'Osiris (eł $\}$ ) du verset (24). Le premier signe $w$ est clair, le sceptre peut se lire 'b3, donc ', et l'oie peut avoir la valeur $h t m$, donc $h$.

C. Catégorie phonétique (2b) : le jeu phonétique suivant n'est pas immédiatement perceptible ; Sauneron fait dériver la valeur phonétique du vase $\mathbb{R}$, figurant dans le nom d'Osiris, du nom d'un récipient, $s n b t^{123}$, qui, sur la colonne d'Esna, se trouve directement au-dessus de la barque, laquelle se lit $w(w i 3>w)$. Pour notre jeu phonétique, nous conservons le $s$ pour le vase tandis que je suggère de lire la barque (e5) imn, une valeur rare, mais néanmoins attestée, pour écrire Amon ou Amon-Rê. En combinant ces éléments, on obtient simn d'où, avec la suppression de la consonne faible, smn. Autrement dit, il s'agit d'un renvoi à l'oie ( du nom d'Osiris du verset (24), qui se lit également smn «oie du Nil».

E. Catégorie allégorique (1a) : le signe $r d(\mho)$, «croître, pousser», renvoie aux plantes- $k 3 k 3$ du texte d'accompagnement.

E. Catégorie allégorique (1a) : j'ai déjà évoqué, dans le commentaire du verset précédent, les relations que l'on pouvait établir entre le crocodile ( étant une forme de Khnoum-Rê, qui poursuit ses ennemis dans l'étang rouge. Les deux pre-

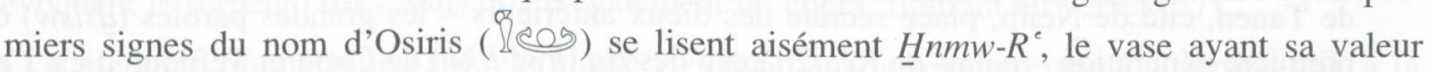
normale $\underline{h} \mathrm{~nm}$ et le disque solaire, sans la barque, $R^{e}$. On aurait donc un renvoi au protagoniste

119 Esna III, 265, 26-27.

120 Voir le verset (29) de la litanie de Menhyt.

121 À propos de la localisation de cette étendue d'eau, voir le commentaire du verset (22); au sujet du toponyme 5 - $d \check{r} r$, en général (entre autres un lac près d'Héliopolis et un lieu de culte d'Hathor) : J. Yoyotte, EPHE 89 (1980-1981), p. 63-65.

122 Au sujet de ce toponyme, on verra J. Yoyotte, RdE 39 (1988), p. 173 et P. Koemoth, WdO 25 (1994), p. 10-12 et récemment D. Meeks, Mythes et légendes du Delta, p. 313 (cf. aussi p. 83-84, n. 203).

123 S. Sauneron, Esna VIII, p. 185, N³66. 
principal - dans une barque (? $)^{124}$ - du combat au moment de la fête de la victoire, le 20 Épiphi. L'épithète hnty ' $33 t-k 3 k 3$ peut, elle aussi, être une désignation de Khoum, seigneur de la campagne $^{125}$. Je n'ai pu trouver une justification pour la place de ce verset dans la litanie $\left(\mathrm{n}^{\circ} 23\right)$; on peut supposer qu'il existe une raison, mais il faut noter que les éléments qui livrent les tertia comparationis sont désormais beaucoup plus rares. En effet, le chiffre 23 rend difficile toute comparaison avec la liste des nomes puisqu'il n'en existe canoniquement que vingt en Basse Égypte et vingt-deux en Haute Égypte. Par conséquent, cette catégorie est, dans la majorité des versets qui suivent, inopérante, à l'exception du $\mathrm{n}^{\circ}$ (30).

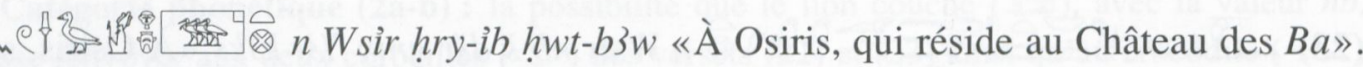
A. Catégorie graphique (2b-c) : le signe $w$ e dans le nom d'Osiris du verset (24) renvoie à une partie de l'œil oudjat ( ), c'est-à-dire de dans le nom d'Osiris du verset (25), mais aussi au crochet de la couronne rouge ( au verset (26) ; une fois sur la tête du second cobra, une autre fois comme élément de la double couronne ( 4 ).

C. Catégorie phonétique (2c) : le sceptre $\hat{\uparrow}$ figurant dans le nom d'Osiris a la valeur normale $s h m$, ce qui donne, par acrophonie, $s$ dans le nom d'Osiris. Avec cette valeur, le signe renvoie à la double couronne $(\mathfrak{y} /)$ du nom d'Osiris du verset (26), qui peut se lire shmty «double couronne».

E. Catégorie allégorique (1a): même si Sauneron n'a rien relevé au sujet d'une relation éventuelle entre le nom d'Osiris et le texte en clair dans le verset (24), on peut quand même mentionner plusieurs textes à Esna qui montrent bien que le sceptre $s h m \hat{\uparrow}$, avec la valeur ' $b 3$, renvoie au toponyme $h w t-b 3 w$. Le premier à permettre de comprendre ce renvoi est la description des cérémonies du 19 Épiphi, gravée sur la colonne 1 d'Esna ${ }^{126}$ : «L'enfer divin qui se trouve à cet endroit, c'est l'enfer mystérieux de Kematef, accompagné de Chou et Tefnout, et d'Atoum qui est enseveli avec eux. C'est l'antre des dieux morts, des sept propos (d Sisw $^{\prime}$ ) matérialisés de Méthyer, en leur aspect de faucons. On les appelle 'dieux antérieurs'. On nomme (cet antre) le château des $B a(h w t-b 3 w)$ pour cette raison. C'est aussi la tombe ('b3) d'Osiris, car c'est là que furent réunis ses membres, à côté de son père, en tant que seigneur de l'Occident.» L'autre texte est gravé sur la même colonne, mais au début de celle-ci ; c'est une description du temple du nord, celui de $P r-n t t^{127}:$ : $P r-n t r$ du dieu des dieux, tombe ( $\left.{ }^{(} b 3\right)$ de Kematef, maison

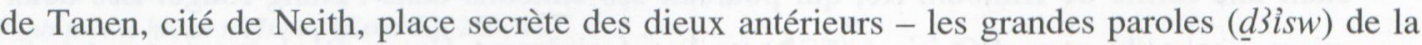
première génération - palais de Rê, Château des $B a(h w t-b 3 w)$ de Chou et Tefnout, c'est l'enfer

124 Suggestion de S. Sauneron, Esna V, p. 375.

125 Esna VI, 519, 14. Dans le même texte (1. 7-9) il est d'abord désigné comme hrry-ib ḩwt-b3w et un peu plus loin comme hnty i3t-k3k3, c'est-à-dire que l'on a peut-être une relation entre les versets (23) et (24) de la litanie d'Osiris.

126 Esna III, 197, 24-25 ; traduction dans Esna V, p. 351. Ce texte est déjà mentionné dans le commentaire du verset (7), cf. supra. Dans le cas présent, il est important de constater qu'il s'agit encore, comme dans les versets (22) et (23), de

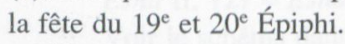

127 Esna III, 196, 2 ; traduction dans Esna v, p. ?.19. 
mystérieux, que nul homme ne saurait voir, l'antre (imht) des dieux morts». Un troisième texte (Esna III, 280A) précise à propos d'Osiris, seigneur de ${ }^{c} b 3$, que $h w t-b 3 w$ est divin grâce à son image.

(25) mm

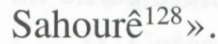

A. Catégorie graphique (1a) : le disque solaire avec le cobra (으) dans le nom d'Osiris renvoie au même signe qui figure dans le toponyme $\operatorname{Pr}-S 3 h w-R^{c}$, dans le texte en clair.

A. Catégorie graphique (1c) : l'œil oudjat ( au même hiéroglyphe sur la main du babouin (

A. Catégorie graphique (2b-c) : le cobra qui s'enroule autour du disque solaire ( $(\bullet)$ ) dans le nom d'Osiris du verset (25) renvoie aux deux cobras (G) dans le nom d'Osiris du verset (26) ainsi qu'aux serpents dans le nom d'Osiris du verset (27).

C. Catégorie phonétique (2b) : l'œil oudjat ( d'Osiris du verset (25) peut aussi faire référence aux deux cobras (ใُ (h) du verset (26), qu'on peut lire w3 $\underline{d} t y$.

C. Catégorie phonétique (2b) : le soleil et la lune ( $(\bullet \bigcirc$ C) dans le nom d'Osiris du verset (25) peuvent se lire snwy «les deux frères» et renvoyer ainsi aux deux déesses, Isis et Nephthys (

E. Catégorie allégorique (1c) : l'œil oudjat ( et celle-ci, qui figure elle-même dans le nom d'Osiris (@) au verset (25), renvoie à Thot, le dieu lunaire, au verset (27).

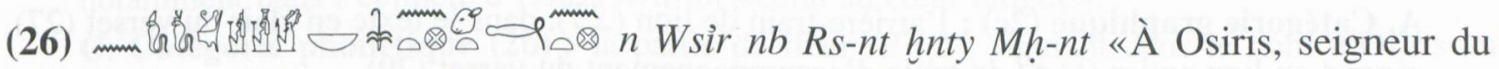
sanctuaire du sud, qui préside au sanctuaire du nord ${ }^{129} »$.

A. Catégorie graphique ( $2 \mathbf{a}$ et $\mathbf{c})$ : la corbeille sur la tête de Nephthys signe dans le texte en clair des versets (26) et (28).

A. Catégorie graphique (2b) : les deux cobras ( 6 ( 6 ) dans le nom d'Osiris du verset (26) évoquent le serpent ( $ァ \Omega)$ ), sans doute également un cobra, figurant au verset (27).

C. Catégorie phonétique (1b) : la plante $s w(\succsim)$, dans le texte en clair du verset (26), a la

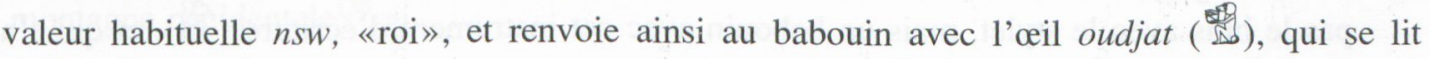
également $n s w$.

128 Une sorte de reposoir au nord d'Esna : S. Sauneron, Esna I, p. 28-29. Celui-ci était consacré à Chou (Esna VI, 495, 3 et 8-9).

129 On trouve chez Ramadan El-Sayed, Documents relatifs à Saïs et ses divinités (BdE 69), 1975, p. 180-199, des remarques au sujet de ces deux sanctuaires de Saïs, où l'on produisait les bandelettes de momie pour Osiris. 
C. Catégorie phonétique (2a): la couronne blanche ( $\$$ ), sur la tête du premier serpent ( n'a pas seulement une lecture $n s w$, mais elle se lit également $\check{s}^{\circ}{ }^{e}$ et elle peut donc être rapprochée des deux valeurs phonétiques courantes de $\succsim$.

C. Catégorie phonétique (2a) : la couronne rouge ( $/ 4$ ), sur la tête du deuxième serpent ( également différentes valeurs ; lue $M h w$, «Basse Égypte», elle renvoie à la première partie du toponyme $m h$ - $n t$ et, avec la valeur $n t$, «couronne rouge», à sa seconde partie.

E. Catégorie allégorique (1a) : les deux couronnes, blanche et rouge, sur la tête des serpents,

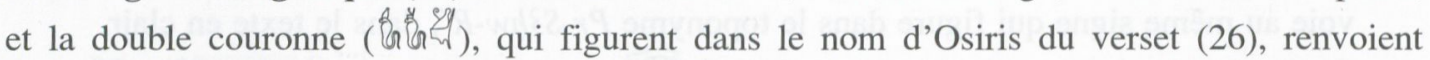
toutes aux deux sanctuaires de Haute et de Basse Égypte à Saïs : le $r s-n t$ et le $m h-n t$ dans le texte en clair.

E. Catégorie allégorique (1a) : les deux déesses, Isis et Nephthys ( d'Osiris, peuvent se lire rhty «les deux compagnes» ${ }^{130}$ et renvoyer ainsi aux deux sanctuaires saïtes de Haute et de Basse Égypte, où ces déesses étaient responsables de la production des étoffes, spécialement des bandelettes servant à envelopper des momies ${ }^{131}$.

E. Catégorie allégorique (1c) : la dernière allusion concerne les quatre points cardinaux. On peut facilement lire $\succsim$, rsy, «sud» et $\stackrel{m}{9}$, mhty, «nord» dans le texte en clair du verset (26)

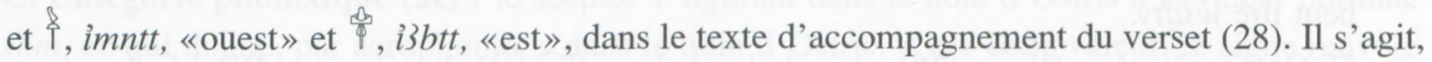
pour les Égyptiens, de l'ordre canonique des point cardinaux : sud, nord, ouest, est ${ }^{132}$.

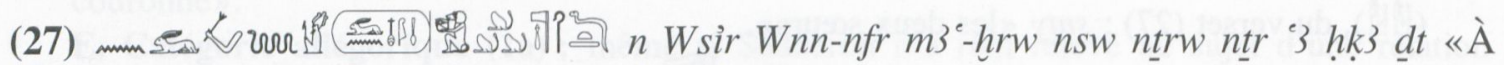
Osiris-Onnophris, juste de voix, roi des dieux, grand dieu, régent de l'éternité $\underline{d} t »$.

A. Catégorie graphique (1a) : le lièvre (SS) renvoie au même signe dans le cartouche.

A. Catégorie graphique (1a) : le groupe 8 rappelle la même combinaison au verset suivant.

A. Catégorie graphique (2c) : l'arrière-train de lion $(\Omega)$, dans le texte en clair du verset (27), répond au lion entier ( 10 ) du texte d'accompagnement du verset (29).

B. Catégorie graphique/phonétique (1a) : cette allusion est plus intéressante. Il s'agit de déterminer l'élément commun entre les trois arrière-trains de lion $(\Omega)$ et le sceptre $h k 3 z(?)$ : c'est la valeur phonétique $h k 3$, une écriture alternative de $h k 3$ étant précisément l'arrière-train de lion sur un étendard ( $\uparrow)$.

D. Catégorie phonétique/allégorique (1a) : le bras avec le stylet $($ ) renvoie par deux fois au dieu Thot. D'une part, il existe une variante de ce signe ayant une lecture $s p h r$, qui ne figure pas le bras avec le stylet, mais un babouin avec cet instrument d'écriture (趝), et qui s'apparente beaucoup au babouin (盟) du texte en clair. D'autre part, le verbe $s p h r$ fait déjà allusion

130 Cf. S. Sauneron, Esna VIII, p. $123, \mathrm{~N}^{\circ} 52,3^{\mathrm{e}}$ possibilité. Pour la lecture rhty (à la place de rhty), voir B. Backes, GM 180 (2001), p. 23-28.

${ }^{131}$ Les références sont nombreuses : Ramadan El-Sayed, loc. cit. ; B. Backes, loc. cit. et Id., Rituelle Wirklichkeit (Rites Égyptiens 9), 2001, p. 77-79.

132 Cf. G. Posener, dans S. Schott (éd.), Göttinger Vorträge (NAWG I), 1965, p. 69-78. 
au dieu Thot, puisque sphr $r$ c'est «copier des annales, recopier, etc.» et qu'il s'agit de l'une des tâches les plus caractéristiques de ce dieu ${ }^{133}$.

D. Catégorie phonétique/allégorique (1a): le lièvre (senvoie également au dieu Thot. En effet, sa ville principale, Hermopolis, se trouvait dans le XVe nome de Haute Égypte ; or, le nom de ce nome était $W n t$, tandis qu'un autre nom d'Hermopolis était $W n w$ ou $W n w$ rsy «l'Hermopolis du sud».

D. Catégorie phonétique/allégorique (1b) : la dernière allusion est la plus difficile à déceler: comment le bras avec le stylet $($ ) peut-il renvoyer à la tête (慨)? En réalité ce n'est pas un problème, il faut simplement lire $s f \underline{h}$ et non pas $s p \underline{h} r$, un verbe qui a plus ou moins le même sens ${ }^{134}$. Le verbe $s f h$ est normalement déterminé par ce bras et, si on trouve ce signe employé comme idéogramme, c'est uniquement par convention qu'on le lit $s p h r$ et non pas $s f h$. Or, $s f h$ c'est aussi le chiffre sept et, la tête comptant sept ouvertures (deux yeux, deux oreilles, deux narines et la bouche), on peut simplement écrire $t p$ avec sept traits et vice-versa.

(28) था préside à l'Occident, le grand dieu, le seigneur d'Abydos».

A. Catégorie graphique (2b) : je ne suis pas convaincu de la réalité de ce premier jeu graphique. La tête (飣) employée dans le nom d'Osiris du verset (28) peut renvoyer à la tête de l'homme assis (1) tout comme à la momie (l) du verset (29).

C. Catégorie phonétique (1b) : l'hirondelle ( $)$, avec sa valeur usuelle $w r$, renvoie naturellement au groupe $w r(\stackrel{\&}{S})$ du texte en clair du verset $(29)$.

C. Catégorie phonétique (1b) : l'hirondelle ( $)$ et la plante $r d(\mho)$ peuvent se lire $w r+r d=$ $w r \underline{d}$, ce qui répond à l'homme agenouillé ( notamment dans l'épithète d'Osiris $w r \underline{d}-i b$, «celui au cœur fatigué».

C. Catégorie phonétique (2b) : la partie de fleur $r d$ (૪), figurant dans le nom d'Osiris au verset (28), renvoie phonétiquement à la pustule $\left(\stackrel{\circ}{ }^{\circ} \mathrm{O}\right)^{135} \mathrm{du}$ verset suivant, dont la lecture est $r d w$, «écoulements».

E. Catégorie allégorique (1a) : pour ce qui relève de la catégorie allégorique, l'exemple de la tête (羿), qui évoque le toponyme $3 b \underline{d} w$, «Abydos», est un des plus clairs ; Sauneron a déjà noté qu'il s'agissait précisément de la relique abydénienne. L'un des textes les plus connus à ce sujet se trouve dans le papyrus Jumilhac (III, 20 - IV, 1, bas) : «Le 19e (jour) du quatrième mois du printemps (c'est-à-dire le 19 Khoiak), c'est le jour où fut trouvée la tête, établie dans la montagne occidentale ${ }^{136}$.»

133 Cf. Chr. Leitz (éd.), $L G G$ VI, $277 \mathrm{a}-278 \mathrm{~b}$.

134 Cf. Chr. Leitz (éd.), LGG VI, 302b-c.

135 L'identité du signe n'est pas assurée : voir la discussion de D. Meeks, Les architraves du temple d'Esna (Paléographie hiéroglyphique 1), 2004, 233-234, §631.

${ }^{136}$ Une autre référence importante se trouve au temple de Séti I ${ }^{\text {er }}$ à Abydos (A. M. Calverley, A. H. Gardiner, The Temple of Sethos I at Abydos, III. The Osiris Complex, 1938, pl. 6) : la tête est ici peinte de face sur un objet qui ressemble à la résille du prétendu fétiche abydénien et qui est désigné comme «Osiris». Pour l'ensemble de cette question, on verra 
E. Catégorie allégorique (1b) : imntt, «ouest», renvoie à la nécropole ( $t 3-\underline{d} s r)$ qui se trouve, bien entendu, à l'ouest ${ }^{137}$.

E. Catégorie allégorique (1b) : le toponyme $3 b d w$, «Abydos», renvoie tout naturellement à $T 3$-wr, «le nome Abydénien», qui, à cette époque, peut également être une simple désignation de la ville d'Abydos.

(29)

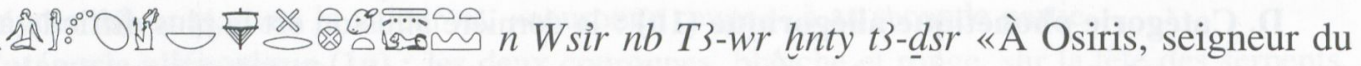
nome thinite, qui préside à la nécropole».

B. Catégorie graphique/phonétique (1a) : l'homme agenouillé (觔) dans le nom d'Osiris renvoie, avec la lecture $w r \underline{d}$, «être fatigué», au groupe $₫$ dans le texte en clair du même verset. C. Catégorie phonétique (2c) : 领 a une deuxième lecture, hms, «s'asseoir», et répond ainsi au signe 4 , dans le nom d'Osiris du verset (31), qui se lit hrmwst.

D. Catégorie phonétique/allégorique (1b) : l'allusion suivante n'est pas, à première vue, évidente. Comment peut-on définir les relations entre la momie (l) et le collier ( $\left.{ }^{\circledR}{ }^{2}\right)$ ? Une lecture possible pour la momie est $m n w$, notamment quand il désigne la statue $(m n w)$. Mais le collier wsh n'a pas du tout cette valeur phonétique. On peut quand même trouver une allusion, en particulier quand on prend en compte un texte extrait d'une scène rituelle de Dendara, qui a pour titre, $\underline{t} s w s h$, «nouer le collier». La formule prononcée par le roi se lit : wsh $n$ mnw $n$ mnw.tn inh.n.f hh $n$ k3.tn «Le collier composé des plantes $m n w$ est pour vos statues, il enserre le cou de vos $k a{ }^{138} \gg$ On ne sait pas définir précisément, d'un point de vue botanique, la nature de ces plantes $m n w$, mais cela n'a aucune importance ici. Ce qui nous intéresse, c'est le fait que le collier wsh est apparemment composé des plantes $m n w$, il s'agit de l'élément qui permet de faire le lien entre la momie et le collier ${ }^{139}$.

E. Catégorie allégorique (1a) : les trois signes dans le nom d'Osiris évoquent $T 3$ - $w r$, «Abydos». L'homme agenouillé ( $\hat{d}$ ), qu'on lit $w r d$, fait immédiatement penser à $w r \underline{d}$-îb, «celui au cœur

H. Beinlich, Die "Osirisreliquien», p. 222-224 avec des commentaires relatifs au prétendu fétiche abydénien ; la relation entre la tête et le VIII ${ }^{\mathrm{e}}$ nome de Haute Égypte est, selon cet auteur, absolument assurée à partir de l'époque Saïte. Voir également M. Stadler, ZÄS 128 (2001), p. 75-76 au sujet du scarabée sorti de la tête d'Osiris et, récemment, D. Meeks, Mythes et légendes du Delta, p. 174.

137 Suggestion de $\mathrm{M}^{\text {me }}$ Victoria Altmann.

138 Dendara II, 91, 5-7.

139 Cf. M. Beaud, dans S. Israelit-Groll (éd.), Studies in Egyptology Presented to Miriam Lichtheim, 1990, p. 59, n. 21 avec un renvoi à Dendara II, 91, 6 et III, 159, 4. Il n'est pas certain qu'il s'agisse vraiment d'une plante bien déterminée (ainsi M. Beaud avec renvoi au Wb II, 71, 17 évoque un hapax). On pourrait aussi admettre qu'il s'agit du lemme situé juste avant dans le $W b(I I, 71,16)$ : mnw comme désignation générale des plantes. La question mériterait d'être examinée plus en détail. On trouve d'autres références chez Er. Graefe, dans U. Verhoeven, Er. Graefe (éd.), Religion und Philosophie im Alten Ägypten. Festgabe für Philippe Derchain (OLA 39), 1991, p. 144-145, qui considère les plantes mnw comme un motclef peu courant dans les scènes rituelles d'offrandes du collier (p. 136). D'une manière générale, cette liste des mots-clefs est le développement d'une méthode importante pour l'analyse des litanies d'Esna; en effet, il y montre de manière convaincante comment on peut renvoyer, avec un simple mot-clef, à un sujet (ici le collier). Dans le cas présent, au verset (30), la méthode fonctionne dans les deux sens : le mot-clef mnw (mentionné indirectement au verset 29) renvoie au sujet «collier» mentionné au verset (30), lequel renvoie à son tour à un autre mot-clef (Héliopolis) au verset (32). 
fatigué», désignation très courante d'Osiris mort ; la momie (l) et la pustule $\left(\stackrel{8}{0}^{\circ}\right), r \underline{d} w$, «écoulements», rappellent cette localité ${ }^{140}$ et sa célèbre nécropole où se trouvait la tombe d'Osiris. On se contentera pour évoquer ce fait bien connu de quelques références : Osiris est appelé, dans une des chapelles sur le toit de Dendara, «la momie auguste à Abydos $\left(s^{\complement} h \breve{s}\right.$ ps $m$ $T 3-w r){ }^{141}$; dans un papyrus de la XXI ${ }^{\mathrm{e}}$ dynastie, «la momie auguste à Bousiris et à Abydos (s $s^{c}$ šps $m \underline{D} d w T$ T3-wr)» ${ }^{142}$; dans le papyrus Jumilhac (IX, 3-5), on mentionne un lieu appelé «la crypte qui met en avant le fatigué de cœur» ( $d w 3$ t shnt $n t$ wrd-ib), décrit comme «la grande crypte d'Osiris, dans laquelle sont enterrées les humeurs du dieu $(r \underline{d} w$-ntr $r) »$.

E. Catégorie allégorique (1b) : Abydos, dans le texte en clair du verset (29), renvoie à sa contrepartie septentrionale, Bousiris, au verset (30). Par ailleurs, les trois toponymes Abydos, Bousiris et Saïs, évoqués aux versets (29) à (31), sont précisément les scènes des trois étapes du rituel funéraire ${ }^{143}$.

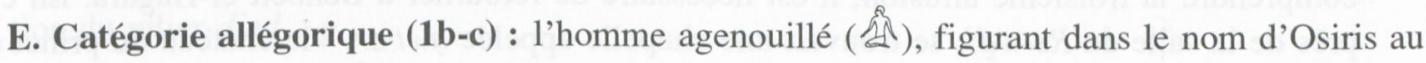
verset (29), avec sa valeur $w r \underline{d}$, fait penser à $w r \underline{d}-i b$, «celui au cœur fatigué», et renvoie également à une autre désignation courante d'Osiris qui figure au verset (30), $r s-w \underline{d} 3$, «celui qui s'éveille en bonne santé», sans doute un autre moment, plus tardif, dans le mythe osirien ${ }^{144}$.

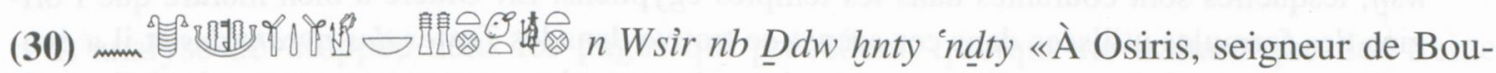
siris, qui préside au nome bousirite ${ }^{145} \gg$.

A. Catégorie graphique (1a) : la première allusion graphique a déjà été relevée par Sauneron, il s'agit des deux piliers djed (10103) dans le nom d'Osiris qui font écho à ceux formant le nom $D d w$, «Bousiris» ${ }^{146}$.

D. Catégorie phonétique/allégorique (1c) : le hiéroglyphe du $\mathrm{IX}^{\mathrm{e}}$ nome de Basse Égypte, ici dans sa variante plus rare ${ }^{147}$, avec un sceptre $h k 3$ (㥀), dans le texte en clair du verset (30) renvoie, avec sa valeur phonétique endty, à la thématique héliopolitaine du verset (32). Héliopolis était la capitale du XIII ${ }^{\mathrm{e}}$ nome de Basse Égypte, dont le nom était $h k{ }^{3}-^{-} n \underline{d}$ : on obtient donc un calembour entre $n \underline{d} t y$ et $h \underline{k} 3^{-}{ }^{i} n \underline{d}$.

${ }_{140}$ Pour les relations des $r \underline{d} w$ et d'Abydos voir maintenant D. Meeks, Mythes et légendes du Delta, p. $6(\S 3=\mathrm{II}, 4)$ et p. $42-44$.

141 Dendara X, 250, 7-8 = Chr. Leitz (éd.), LGG VI, 198a.

142 P. BM 10299, publié par R. A. Caminos, MDAIK 16 (1958), p. 21, pl. 1, 1. 2 = Chr. Leitz (éd.), LGG VI, 198 b.

143 Voir en général H. Altenmüller, dans $L A ̈$ I, col. 42-47, s.v. «Abydosfahrt»; col. 745-765, s.v. «Bestattungsritual» et col. 884, s.v. «Busiris, Fahrt nach».

144 Cf. B. van de Walle, ZÄS 98 (1972), p. 140-149.

145 Pour 'ndty, voir en dernier lieu O. Perdu, RdE 56 (2005), p. 129-166.

146 On trouve une autre représentation de la barque d'Osiris avec deux piliers djed chez A. Fakhry, Bahria Oasis, 1942, pl. 58b (réf. de Françoise Labrique). Les relations entre le pilier djed et la ville de Bousiris sont bien établies depuis le Moyen Empire : H. Altenmüller, dans $L \ddot{A} \mathrm{I}$, col. 1101, s.v. «Djed-Pfeiler»; et la procession géographique à l'extérieur du sanctuaire du temple d'Edfou, où l'on dit à propos du IX nome de Basse Égypte $(E d f o u ~ I, 332,9)$ : «Il (= le roi) t'amène le IX ${ }^{\mathrm{e}}$ nome de Basse Égypte ('ndty), la maison d'Osiris, le seigneur de Bousiris ( $\underline{D} d w)$ avec le pilier djed auguste (dd šps). Le pilier djed y unit les membres divins comme Osiris, seigneur de Bousiris (Ddw)» : H. Beinlich, Die «Osirisreliquien», p. 252.

147 Voir les graphies réunies par H. Gauthier, DG I, p. 151-152. 
E. Catégorie allégorique (1a) : le signe $\mathbb{q} \int \mathbb{\Psi}, r s-w d$, dans le nom d'Osiris, fait référence aux quatre piliers djed du même verset. La compréhension de cette allusion n'est pas évidente au premier abord. Cependant, il faut noter qu'au temple d'Isis à Behbeit el-Hagara, il y avait, selon la reconstitution proposée par Christine Favard-Meeks, une salle qui s'appelait hwt-Rs$w \underline{d} 3$, «le château de $R s-w \underline{d} 3$ ». Un relief en provenant figure une scène rituelle présentant le roi en adoration devant Osiris-Djed ${ }^{148}$; on en déduit qu'il devait exister une relation entre $R s$-wd 3 et le pilier djed.

E. Catégorie allégorique (1a-c) : $r s-w d s$ ( $(0$ f ) renvoie également aux trois localités où cette divinité était vénérée : Bousiris d'abord ${ }^{149}$; Saïs ensuite, où $R s-w d \underline{d 3}$ possédait un temple ${ }^{150}$ - ville dont le nom est mentionné dans le texte en clair du verset (31) - et, enfin, hwt-sr, «le château du prince», un sanctuaire bien connu d'Héliopolis, mentionné au verset (32). Pour comprendre la troisième allusion, il est nécessaire de retourner à Behbeit el-Hagara. En effet, près de la salle du $R s-w d \underline{d} 3$, se trouvait une chapelle appelée $h w t-s r$, «le château du prince» ${ }^{151}$. E. Catégorie allégorique (1a-c) : la dernière allusion de cette catégorie allégorique se cache encore dans le collier 尔 ${ }^{\circledR}$, qui évoque clairement Héliopolis, le sujet du verset (32). J'ai déjà mentionné les deux études de M. Beaud et de Er. Graefe traitant des scènes rituelles du collier wsh, lesquelles sont courantes dans les temples égyptiens. Er. Graefe a bien montré que l'origine des formules utilisées dans ces scènes se trouve dans les Textes des pyramides et il a évoqué des «mots clefs», que l'on retrouve dans chaque scène, comme, par exemple, l'Ennéade d'Héliopolis et le chiffre neuf ${ }^{152}$. Les occurrences où l'on fait allusion au chiffre neuf sont très nombreuses ; on relève aussi que souvent, mais pas systématiquement, dans les représentations, les rangs des colliers sont au nombre de neuf ${ }^{153}$. Ce chiffre en égyptien se dit $p s \underline{d}$, homonyme d'un mot $p s \underline{d}$, «dos, épine dorsale»; or, le pilier djed peut être considéré lui-même comme l'épine dorsale d'Osiris (comme l'indique clairement le chapitre 155 du Livre des morts ${ }^{154}$ ). On peut en conclure que le collier $\left.w s h{ }^{\circledR}{ }^{\circledR}{ }^{\circledR}\right)$, employé dans le nom d'Osiris, renvoie non seulement

148 Chr. Favard-Meeks, Le temple de Behbeit el-Hagara (BSAK 6), 1991, p. 141 et 349-351 et maintenant D. Meeks, Mythes et légendes du Delta, p. 25 (\$27).

$149 \mathrm{Cf}$. Chr. Leitz (éd.), $L G G \mathrm{IV}, 715 \mathrm{a}(R s-w d \underline{1}-m-\underline{D} d w$ «qui s’éveille en bonne santé à Bousiris» comme désignation d'Osiris sur un groupe statuaire de la collection Sabattier [N॰2] = G. Legrain, RT 14 [1893], p. 55).

150 Chr. Leitz (éd.), LGG IV, 714a (F. H. Haikal, Two Hieratic Papyri of Nesmin [BAE XIV], 1970, p. 79 [col. 111, 37] = J.-Cl. Goyon, BIFAO 65 [1967], p. 152). Voir aussi M. Zecchi, A Study of the Egyptian God Osiris Hemag (ASCEVOA 1), 1996, p. $80-81$.

151 Chr. Favard-Meeks, op. cit., p. 303-304 (pour les chapelles du toit) et p. 345-351 (notamment pour la relation potentielle entre Osiris- 'ndty et le $s r$ héliopolitain) et pl. 1 (plan).

${ }_{152}$ M. Beaud, op. cit., p. 46-62. La conception remonte, comme le relève Beaud (p. 46-47, n. 2), au plus tard au Nou-

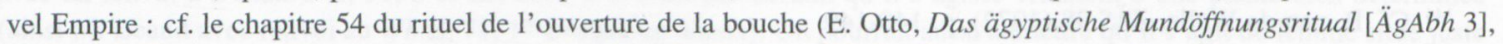
1960, vol. II, p. 119-120) qui trouve son origine dans le chapitre 600 des Textes des pyramides. Cette question est traitée plus précisément par Er. Graefe, op. cit., p. 129-148, où l'on trouve également un tableau qui exploite les 49 scènes rituelles de l'époque gréco-romaine, celles-ci contiennent de nombreux mots-clefs et références à la relation entre le collier et Héliopolis.

153 Cf. Er. Graefe, op. cit., p. 136 et D. Kurth, Edfou VII, 2004, p. 215, n. 4.

154 Indiqué par H. Altenmüller, LÄ I, col. 1102, s.v. «Djed-Pfeiler». D'autres références chez H. Beinlich, Die «Osirisreliquien» ( $\ddot{A} g A b h 42$ ), 1984, p. 252-254 (au sujet des relations entre le dos, le pilier djed et le IX ${ }^{\mathrm{e}}$ nome de Basse Égypte) et D. Meeks, Mythes et légendes du Delta, p. 264, n. 714. 


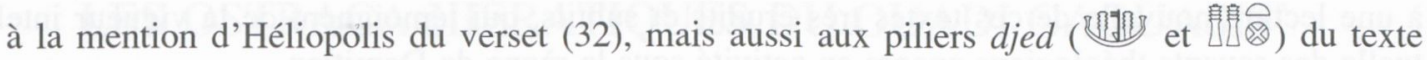
d'accompagnement du verset (30).

F. Relation avec la litanie entière (1a) : la dernière allusion prise en compte pour cette étude préliminaire est un de ces cas où l'on peut connaître la raison secrète qui justifie la position de ce verset dans la litanie. La solution est aussi simple qu'étonnante. Avec la mention de ces quatre piliers-djed au verset (30), on fait allusion à l'érection du pilier djed, cérémonie qui se déroulait le quatrième mois de l'année, le trentième jour. La tradition établissant cette fête ce jour-là est anciennement établie. Le texte le plus explicite se trouve sans doute dans les Mystères d'Osiris au mois de Khoiak, célèbre document situé sur le toit du temple de Dendara ${ }^{155}$ : «Quant au dernier jour de Khoiak, érection du pilier-djed à Bousiris, ce jour de l'enterrement d'Osiris au lieu des arbres $n b h$, dans la caverne qui est sous les arbres $i \check{s} d$, parce que c'est en ce jour que furent amenés les divins membres d'Osiris, après l'ensevelissement d'Osiris. Érection du pilier-djed...»

\section{Conclusion}

L'analyse développée dans les pages qui précèdent, quoique ne traitant que des trente premiers versets de la litanie d'Osiris, a permis de montrer que le commentaire de ces hymnes, créés au tournant du premier et du deuxième siècle avant notre ère, n'en est encore qu'à ses prémices. Ceux-ci constituent une véritable «œuvre d'art» linguistique et graphique qui, avec un nombre assez limité de hiéroglyphes, offre au hiérogrammate érudit la possibilité de créer des quantités incommensurables d'associations et d'allusions mythologiques. On pourrait assimiler cette somme de textes à une sorte de hieroglyphica qui, à travers Horapollon et d'autres auteurs grecs, a influencé largement l'image de l'Égypte pendant la Renaissance et les époques postérieures ${ }^{156}$.

\section{Résumé / Abstract}

Dans cette contribution nous avons développé une nouvelle analyse des trente premiers versets d'une litanie adressée à Osiris $\left(E s n a \mathrm{n}^{\circ} 217\right)$ dont S. Sauneron, dans le commentaire publié après sa mort prématurée (Esna VIII, p. 76-77), n'avait pu mettre en avant la structure complète. Une des clefs permettant de comprendre la structure de ce texte consiste à prendre en compte et analyser non seulement les relations qui existent entre les différentes graphies, souvent très élaborées, du nom d'Osiris et les gloses qui les accompagnent (ce que Sauneron avait déjà fait), mais aussi à examiner les relations qui peuvent être déterminées entre les versets eux-mêmes. Tout ceci conduit

155 É. Chassinat, Le mystère d'Osiris au mois de Khoiak, 1966-1968, p. 756. Pour la traduction «en dehors de» ( $m$ rwty) cf. 1. 130 du même texte (É. Chassinat, op. cit., p. 773).

156 Cf. en dernier lieu J. Assmann, Erinnertes Ägypten, 2006, p. 45-74. 
à une lecture nouvelle de ces textes très érudits et subtils, qui témoignent de la vigueur intellectuelle des savants théologiens encore en activité sous le règne de Domitien.

This article develops a new analysis of the first thirty verses of a litany to Osiris (Esna $\mathrm{n}^{\circ} 217$ ) the whole structure of which S. Sauneron was unable to present in the commentary published after his untimely death. One of the clues for understanding the structure of this text is to consider not only the relations between the often quite sportive writings of the name of Osiris and the accompaning text within a single paragraph (which S. Sauneron already did), but also to examine the relations between one paragraph and the next two. This allowed a new reading of this sophisticated text and shows the great intellectual vigour of the Egyptian priests even in the time of Domitian. 